\title{
'Ionic crystals' consisting of trinuclear macrocations and polyoxometalate anions exhibiting single crystal to single crystal transformation: breathing of crystals ${ }^{\dagger}$
}

\author{
T ARUMUGANATHAN ${ }^{\mathrm{a}}$, ASHA SIDDIKHA $^{\mathrm{b}}$ and SAMAR K DAS ${ }^{\mathrm{b}, *}$ \\ ${ }^{a}$ Department of Chemistry, Thiagarajar College, Madurai 625 009, Tamilnadu, India \\ ${ }^{\mathrm{b}}$ School of Chemistry, University of Hyderabad, Hyderabad, Telangana 500 046, India \\ E-mail: skdas@uohyd.ac.in
}

MS received 30 March 2017; revised 25 May 2017; accepted 9 June 2017

\begin{abstract}
Ion pairing of trinuclear macrocation cluster (known as basic carboxylate), $\left[\mathrm{M}_{3}\left(\mu_{3^{-}}\right.\right.$ $\left.\mathrm{O})\left(\mathrm{ClCH}_{2} \mathrm{COO}\right)_{6}\left(\mathrm{H}_{2} \mathrm{O}\right)_{3}\right]^{1+}$ and a Keggin type polyoxometalate cluster anion $\left[\mathrm{SiW}_{12} \mathrm{O}_{40}\right]^{4-}$ is stabilized with a number of crystal water molecules in composite type compounds $\left[\mathrm{M}_{3}\left(\mu_{3}-\mathrm{O}\right)\left(\mathrm{ClCH}_{2} \mathrm{COO}\right)_{6}\left(\mathrm{H}_{2} \mathrm{O}\right)_{3}\right]_{4}$ $\left[\mathrm{SiW}_{12} \mathrm{O}_{40}\right] \cdot \mathrm{xH}_{2} \mathrm{O} \cdot 2 \mathrm{ClCH}_{2} \mathrm{COOH}\left[\mathrm{M}=\mathrm{Fe}^{3+}, \mathrm{x}=18(\mathbf{1}) ; \mathrm{M}=\mathrm{Cr}^{3+} \mathrm{x}=14(2)\right]$. When the crystals of 1 are heated at $85^{\circ} \mathrm{C}$ and $135^{\circ} \mathrm{C}$ for 3.5 hours in an open atmospheric condition, it goes to $\left[\mathrm{Fe}_{3}\left(\mu_{3}-\right.\right.$ $\left.\mathrm{O})\left(\mathrm{ClCH}_{2} \mathrm{COO}\right)_{6}\left(\mathrm{H}_{2} \mathrm{O}\right)_{3}\right]_{4}\left[\mathrm{SiW}_{12} \mathrm{O}_{40}\right] \cdot 10 \mathrm{H}_{2} \mathrm{O} \cdot 2 \mathrm{ClCH}_{2} \mathrm{COOH}$ (dehydrated 1-85 $\left.\equiv \mathbf{1}^{\prime}\right)$, and $\left[\mathrm{Fe}_{3}\left(\mu_{3}-\right.\right.$ $\left.\mathrm{O})\left(\mathrm{ClCH}_{2} \mathrm{COO}\right)_{6}\left(\mathrm{H}_{2} \mathrm{O}\right)_{3}\right]_{4}\left[\mathrm{SiW}_{12} \mathrm{O}_{40}\right] \cdot 8 \mathrm{H}_{2} \mathrm{O} \cdot 2 \mathrm{ClCH}_{2} \mathrm{COOH}$ (dehydrated 1-135 $\left.{ }^{\circ} \equiv \mathbf{1}^{\prime \prime}\right)$ respectively with the loss of considerable amount of lattice water molecules retaining their single crystallinity. On the other hand, the single crystals of compound 2 , upon heating at $85^{\circ} \mathrm{C}$ or $135^{\circ} \mathrm{C}$ for 3.5 hours, undergo 'crystal-to-crystal transformation' to the single crystals of $\left[\mathrm{Cr}_{3}\left(\mu_{3}-\mathrm{O}\right)\left(\mathrm{ClCH}_{2} \mathrm{COO}\right)_{6}\left(\mathrm{H}_{2} \mathrm{O}\right)_{3}\right]_{4}\left[\mathrm{SiW}_{12} \mathrm{O}_{40}\right] \cdot 8 \mathrm{H}_{2} \mathrm{O} \cdot 2 \mathrm{ClCH}_{2} \mathrm{COOH}$ (dehydrated $\mathbf{2} \equiv \mathbf{2}^{\prime}$ ). Crystal structure analyses show that the parent compounds $\mathbf{1}$ and $\mathbf{2}$ undergo molecular rearrangement (molecular motion in the solid state) in respective dehydrated compounds. Remarkably, these dehydrated crystals $\left(\mathbf{1}^{\prime}, \mathbf{1}^{\prime \prime}\right.$ and $\left.\mathbf{2}^{\prime}\right)$, upon exposure to water vapor at an ambient condition, regenerate the crystals of parent compounds $\mathbf{1}$ and $\mathbf{2}$, respectively.
\end{abstract}

Keywords. Iron and chromium basic carboxylates; silicotungstate Keggin cluster anion; ion pairing into ionic crystals; single crystal to single crystal transformations; dehydration; rehydration.

\section{Introduction}

Transition metal carboxylate chemistry, in particular, trinuclear oxo-centered macrocation, generally formulated as $\left[\mathrm{M}_{3} \mathrm{O}(\mathrm{OOCR})_{6} \mathrm{~L}_{3}\right]^{+}$(where $\mathrm{M}=\mathrm{Fe}^{3+}$, $\mathrm{Cr}^{3+}$, etc., $\mathrm{R}=\mathrm{H}, \mathrm{CH}_{3}, \mathrm{CH}_{2} \mathrm{Cl}$, etc., $\mathrm{L}=$ neutral ligands, such as, $\mathrm{H}_{2} \mathrm{O}$, pyridine, etc.), has played vital role in the development of modern inorganic chemistry. ${ }^{1-5}$ The $\left\{\mu_{3}-\mathrm{O}\right\}$-centered trinuclear metal complexes are also known as 'basic' metal carboxylate complexes. Two basic carboxylate complexes, $\left[\mathrm{Fe}_{3}\left(\mu_{3}-\mathrm{O}\right)\left(\mathrm{OOCCH}_{3}\right)_{6}\left(\mathrm{H}_{2} \mathrm{O}\right)_{3}\right] \mathrm{Cl} \cdot 6 \mathrm{H}_{2} \mathrm{O}$ and $\left[\mathrm{Cr}_{3}\left(\mu_{3}-\right.\right.$ O) $\left.\left(\mathrm{OOCCH}_{3}\right)_{6}\left(\mathrm{H}_{2} \mathrm{O}\right)_{3}\right] \mathrm{Cl} \cdot 6 \mathrm{H}_{2} \mathrm{O}$, whose physical properties have been exhaustively studied, are known for

\footnotetext{
*For correspondence

$\dagger$ Dedicated to Professor K. C. Kumara Swamy on the occasion of his 60th birth anniversary.
}

over 50 years. ${ }^{6}$ An interesting feature of a homonuclear 'basic carboxylate complex' (when the concerned metal centers are paramagnetic) is that the complex nearly always fails to adopt the expected 3 -fold symmetry. ${ }^{6-8}$ It has been argued that net antiferromagnetic coupling distorts spontaneously to a non-3-fold symmetry as a result of spin frustration (also known as "magnetic Jahn Teller effect"). ${ }^{9}$ We have been working on basic trinuclear cluster containing compounds and exploiting their solid state properties to obtain new materials in application point of view. Recently, we have demonstrated that the basic iron trinuclear $\left(\mu_{3}-\mathrm{O}\right)$ cluster-containing compound is capable of sensing methanol in a single crystal to single crystal transformation study. ${ }^{10} \mathrm{We}$ have also shown that the same iron basic carboxylate can recognize pyridine molecule from its vapor state in a gas-solid reaction. ${ }^{11}$ Generally, basic trinuclear 
Table 1. Crystal Data and Structural Refinement Parameters for Compound 1, dehydrated1' and dehydrated 1".

\begin{tabular}{|c|c|c|c|}
\hline & 1 & $1^{\prime}$ & $1^{\prime \prime}$ \\
\hline Empirical formula & $\mathrm{C}_{52} \mathrm{H}_{114} \mathrm{Cl}_{26} \mathrm{Fe}_{12} \mathrm{O}_{126} \mathrm{SiW}_{12}$ & $\mathrm{C}_{52} \mathrm{H}_{98} \mathrm{Cl}_{26} \mathrm{Fe}_{12} \mathrm{O}_{118} \mathrm{SiW}_{12}$ & $\mathrm{C}_{52} \mathrm{H}_{94} \mathrm{Cl}_{26} \mathrm{Fe}_{12} \mathrm{O}_{116} \mathrm{SiW}_{12}$ \\
\hline Formula weight & 6581.62 & 6437.49 & 6401.46 \\
\hline $\mathrm{T}[\mathrm{K}]$ & $293(2)$ & $293(2)$ & $293(2)$ \\
\hline$\lambda[\AA]$ & 0.71073 & 0.71073 & 0.71073 \\
\hline Crystal system & Monoclinic & Monoclinic & Monoclinic \\
\hline Space group & $C 2 / c$ & $C 2 / c$ & $C 2 / c$ \\
\hline$a[\AA]$ & $30.283(9)$ & $30.607(8)$ & $30.507(13)$ \\
\hline$b[\AA]$ & $17.759(5)$ & $17.805(5)$ & $17.764(7)$ \\
\hline$c[\AA]$ & $32.435(9)$ & $30.083(8)$ & $29.939(12)$ \\
\hline$\alpha[\mathrm{deg}]$ & 90.0000 & 90.000 & 90.000 \\
\hline$\beta[\mathrm{deg}]$ & $101.292(4)$ & $113.060(4)$ & $112.941(7)$ \\
\hline$\gamma[\mathrm{deg}]$ & 90.0000 & 90.000 & 90.000 \\
\hline$V\left[\AA^{3}\right]$ & $17106(8)$ & $15084(7)$ & 14941(11) \\
\hline$Z$ & 4 & 4 & 4 \\
\hline Dcalc $\left[\mathrm{Mg} \mathrm{m}^{-3}\right]$ & 2.556 & 2.835 & 2.846 \\
\hline$\mu\left[\mathrm{mm}^{-1}\right]$ & 9.536 & 10.806 & 10.908 \\
\hline $\mathrm{F}[000]$ & 12360 & 12040 & 11960 \\
\hline Crystal size $\left[\mathrm{mm}^{3}\right]$ & $0.36 \times 0.08 \times 0.06$ & $0.28 \times 0.16 \times 0.04$ & $0.18 \times 0.10 \times 0.06$ \\
\hline$\theta$ range for data collection $[\mathrm{deg}]$ & 1.28 to 26.00 & 1.35 to 28.37 & 1.40 to 26.16 \\
\hline Reflections Collected/unique & $87670 / 16902$ & $85120 / 17989$ & $74774 / 14813$ \\
\hline $\mathrm{R}$ [int] & 0.0570 & 0.0676 & 0.1314 \\
\hline Refinement method & Full-matrix least-squares on $\mathrm{F}$ & & \\
\hline Data/restraints/parameters & $16902 / 0 / 1009$ & $17989 / 0 / 1015$ & $14813 / 0 / 953$ \\
\hline Goodness-of-fit on F2 & 1.164 & 1.076 & 0.963 \\
\hline $\mathrm{R} 1 / \mathrm{wR} 2[\mathrm{I}>2 \sigma(\mathrm{I})]$ & $0.0503 / 0.1216$ & $0.0512 / 0.0991$ & $0.0531 / 0.1149$ \\
\hline R1/wR2 (all data) & $0.0596 / 0.1278$ & $0.0706 / 0.1056$ & $0.1028 / 0.1333$ \\
\hline $\begin{array}{l}\text { Largest diff. Peak/hole } \\
{\left[\mathrm{e} \AA^{-3}\right]}\end{array}$ & $1.978 /-2.314$ & $1.862 /-1.668$ & $2.074 /-1.46$ \\
\hline
\end{tabular}
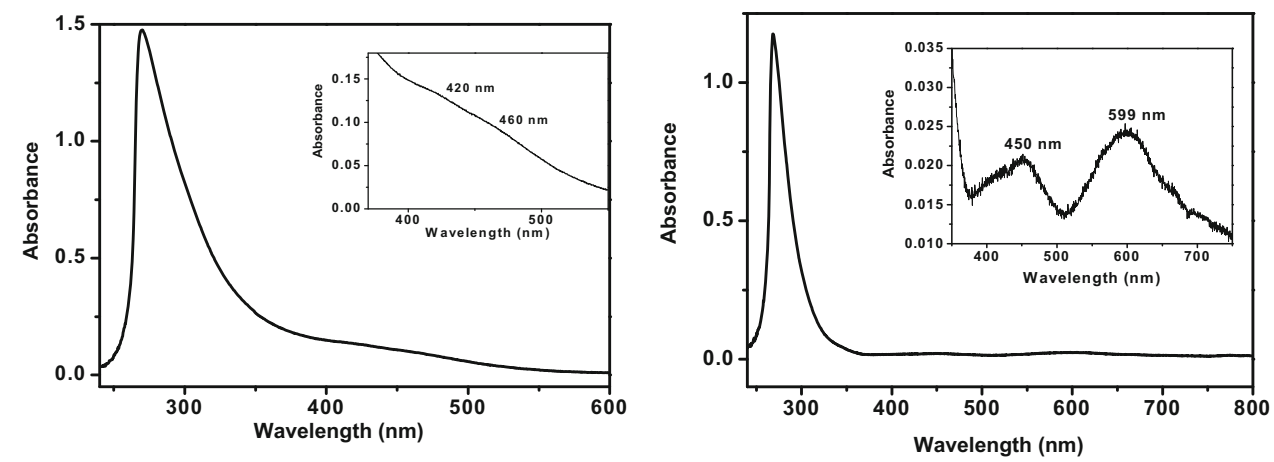

Figure 1. UV-Visible absorbance spectra of, (left) $\left[\mathrm{Fe}_{3}\left(\mu_{3}-\mathrm{O}\right)\left(\mathrm{ClCH}_{2} \mathrm{COO}\right)_{6}\left(\mathrm{H}_{2} \mathrm{O}\right)_{3}\right]_{4}$ $\left[\mathrm{SiW}_{12} \mathrm{O}_{40}\right] \cdot 18 \mathrm{H}_{2} \mathrm{O} \cdot 2 \mathrm{ClCH}_{2} \mathrm{COOH}(\mathbf{1})$ and (right) $\left[\mathrm{Cr}_{3}\left(\mu_{3}-\mathrm{O}\right)\left(\mathrm{ClCH}_{2} \mathrm{COO}\right)_{6}\left(\mathrm{H}_{2} \mathrm{O}\right)_{3}\right]_{4}$ $\left[\mathrm{SiW}_{12} \mathrm{O}_{40}\right] \cdot 14 \mathrm{H}_{2} \mathrm{O} \cdot 2 \mathrm{ClCH}_{2} \mathrm{COOH}(2)$ in $1 \times 10^{-4} \mathrm{M}$.in methanol solution. For compound 1: $\lambda_{\max }(\mathrm{nm})=460,420$ and 270 and corresponding $\varepsilon_{\max }\left(\mathrm{M}^{-1} \mathrm{~cm}^{-1}\right)=924,1326$ and 14510, respectively. For compound 2: $\lambda_{\max }(\mathrm{nm})=599,450$ and 268 and corresponding $\varepsilon_{\max }\left(\mathrm{M}^{-1} \mathrm{~cm}^{-1}\right)=242,211$ and 11660 , respectively.

$\left(\mu_{3}-\mathrm{O}\right)$-bridged transition metal carboxylate clusters are well documented in the literature in three different contexts: (i) discrete $\left(\mu_{3}-\mathrm{O}\right)$ clusters, that are classified either under neutral mixed metal $-\left(\mu_{3}-\mathrm{O}\right)$ clusters or discrete cationic $\left(\mu_{3}-\mathrm{O}\right)$ clusters. ${ }^{6}$ These basic trin- uclear clusters are considered as important subclass of inorganic trinuclear compounds because of their numerous applications such as, majority of higher nuclear clusters \{e.g., $\mathrm{Fe}_{10}$ wheel type clusters) are generated from these starting precursors. ${ }^{12,13}$ This class of com- 

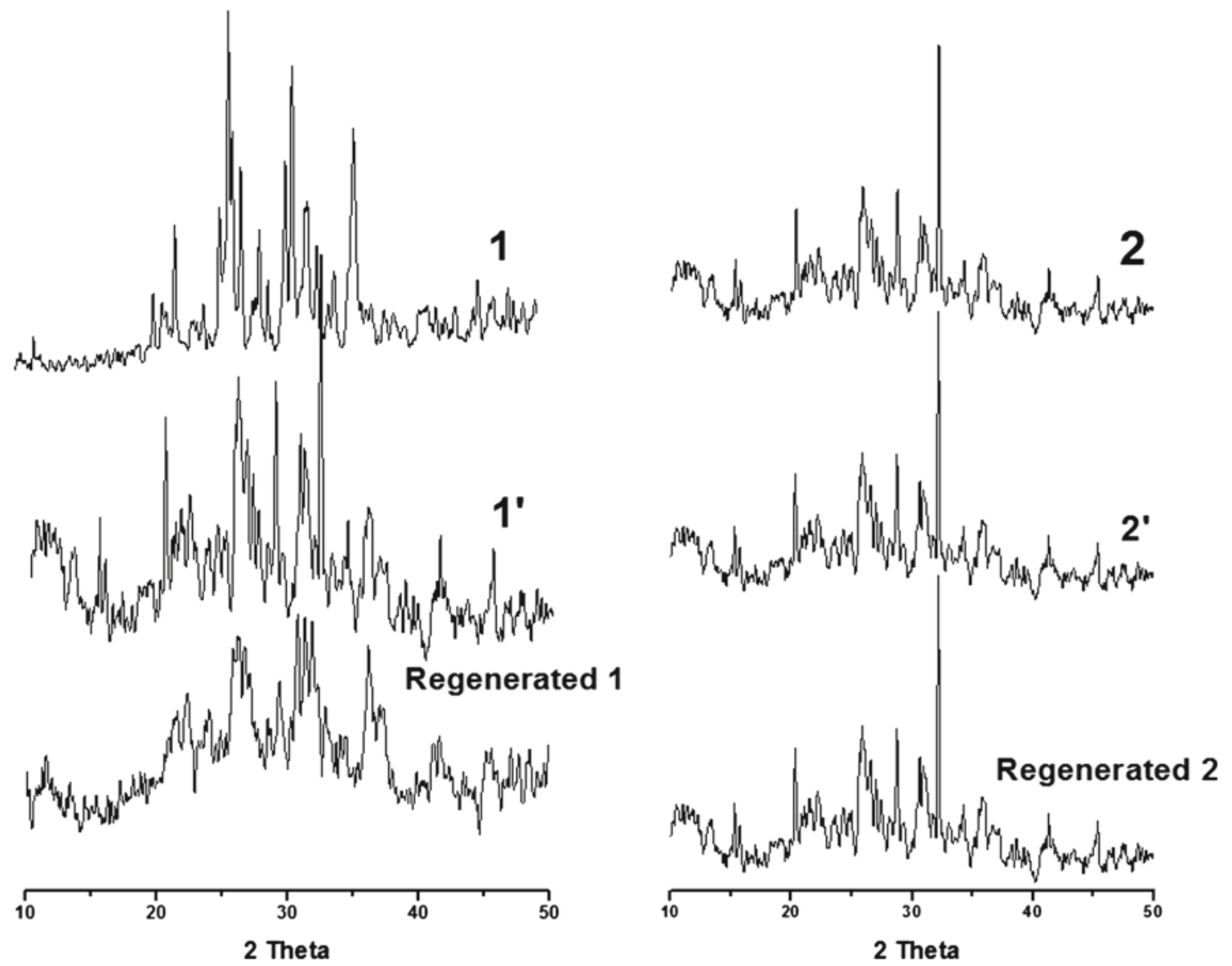

Figure 2. PXRD patterns of basic carboxylate-Keggin based ionic crystals. left: compounds $\mathbf{1}, \mathbf{1}^{\prime}$ and regenerated 1; right: $2,2^{\prime}$ (dehydrated at $85^{\circ} \mathrm{C}$ ) and regenerated 2 .
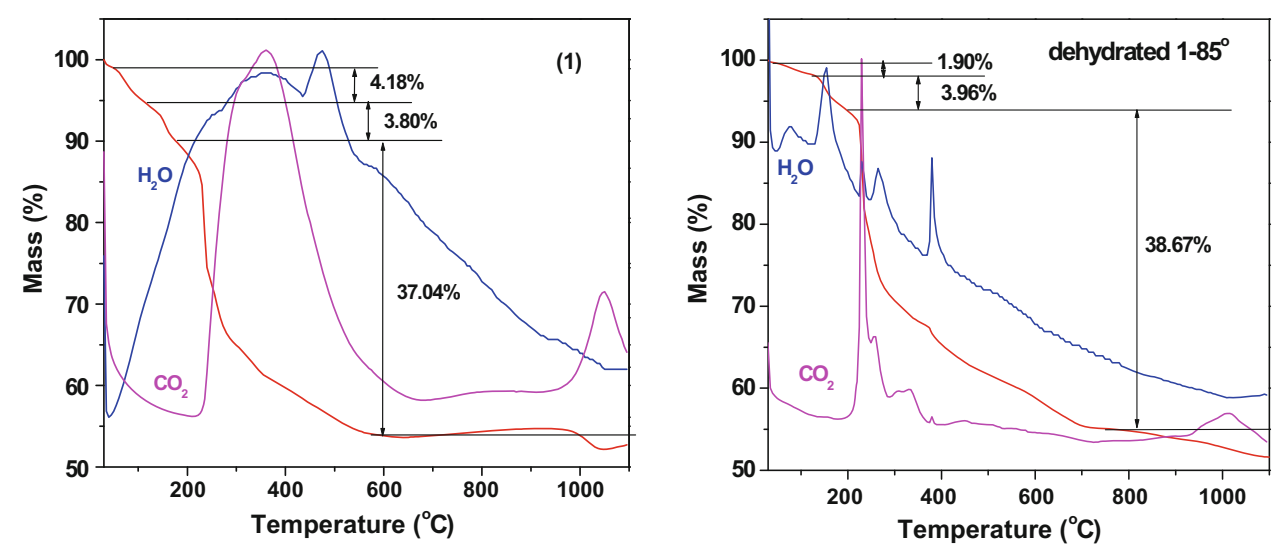

Figure 3. TGA/Mass plot of $\left[\mathrm{Fe}_{3}\left(\mu_{3}-\mathrm{O}\right)\left(\mathrm{ClCH}_{2} \mathrm{COO}\right)_{6}\left(\mathrm{H}_{2} \mathrm{O}\right)_{3}\right]_{4}\left[\mathrm{SiW}_{12} \mathrm{O}_{40}\right] \cdot 18 \mathrm{H}_{2} \mathrm{O} \cdot 2$ $\mathrm{ClCH}_{2} \mathrm{COOH}(\mathbf{1})$ (left); TGA/Mass plot of $\left[\mathrm{Fe}_{3}\left(\mu_{3}-\mathrm{O}\right)\left(\mathrm{ClCH}_{2} \mathrm{COO}\right)_{6}\left(\mathrm{H}_{2} \mathrm{O}\right)_{3}\right]_{4}\left[\mathrm{SiW}_{12} \mathrm{O}_{40}\right]$. $10 \mathrm{H}_{2} \mathrm{O} \cdot 2 \mathrm{ClCH}_{2} \mathrm{COOH}\left(\mathbf{1}^{\prime}\right)$ (right).

pounds are also important in terms of magnetism, ${ }^{14}$ and catalysis. ${ }^{15}$

(ii) Open metal-organic framework materials (MOFs), in which trinuclear $\left(\mu_{3}-\mathrm{O}\right)$-clusters, acting as building units, are interconnected by organic linkers using covalent bonds in the relevant crystal lattice having well-defined pores and cavities. ${ }^{16}$ (iii) Ionic crystals, in which 's' block metal ions (e.g., $\mathrm{Na}^{+}, \mathrm{K}^{+}, \mathrm{Rb}^{+}$, $\mathrm{Cs}^{+}$ions), trinuclear macrocations, polyoxometalate anions and solvent molecules are accommodated in the crystal lattice and stabilized by electrostatic inter actions. ${ }^{17-25}$

Polyoxometalates are metal-oxide based molecular clusters, generally observed in anionic forms, that 

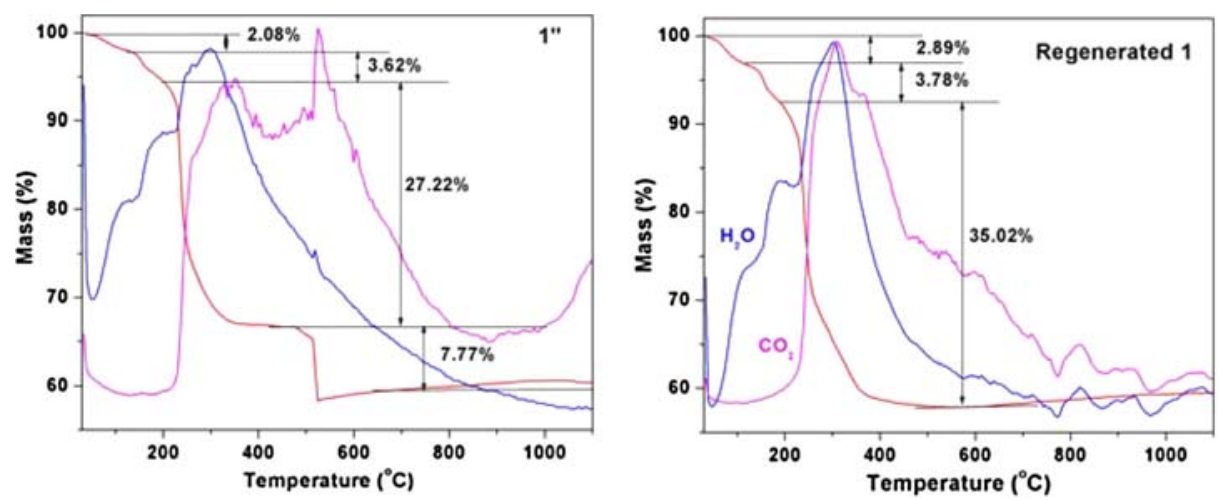

Figure 4. TGA/Mass plot of $\left[\mathrm{Fe}_{3}\left(\mu_{3}-\mathrm{O}\right)\left(\mathrm{ClCH}_{2} \mathrm{COO}\right)_{6}\left(\mathrm{H}_{2} \mathrm{O}\right)_{3}\right]_{4}\left[\mathrm{SiW}_{12} \mathrm{O}_{40}\right] \cdot 8 \mathrm{H}_{2} \mathrm{O} \cdot 2$ $\mathrm{ClCH}_{2} \mathrm{COOH}\left(\mathbf{1}^{\prime \prime}\right)$ (left); TGA/Mass plot of water regenerated compound (regenerated 1) (right).
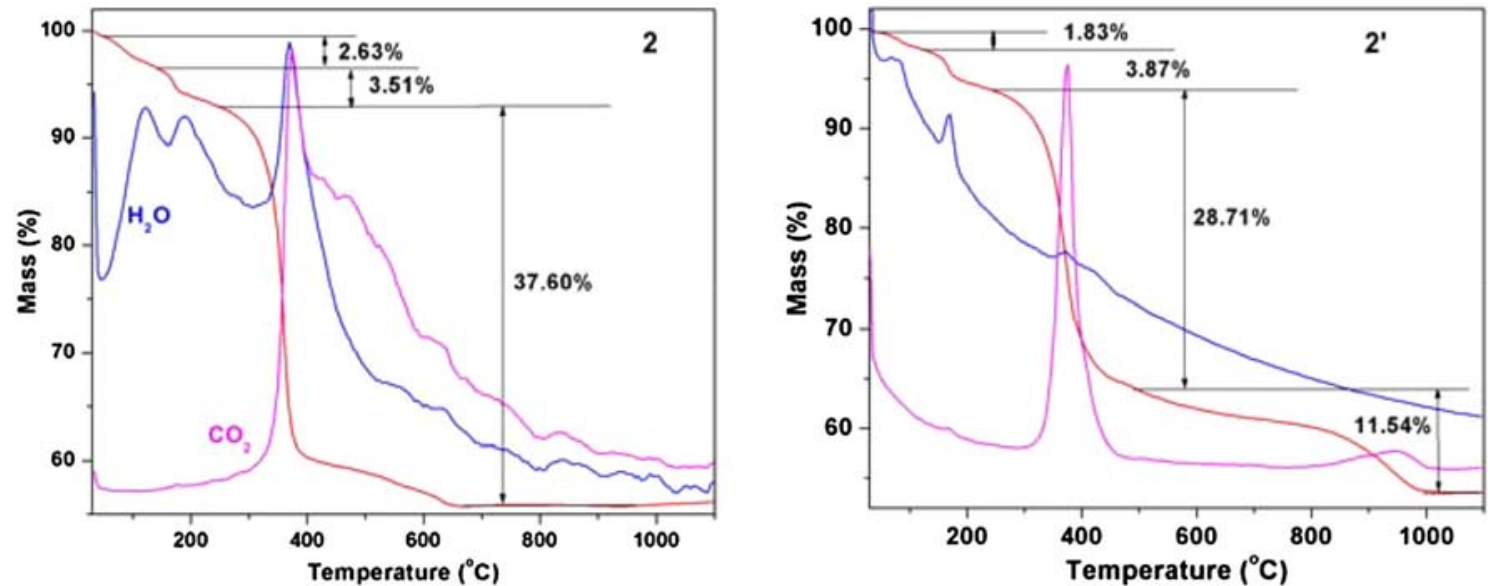

Figure 5. TGA/Mass plot of $\left[\mathrm{Cr}_{3}\left(\mu_{3}-\mathrm{O}\right)\left(\mathrm{ClCH}_{2} \mathrm{COO}\right)_{6}\left(\mathrm{H}_{2} \mathrm{O}\right)_{3}\right]_{4}\left[\mathrm{SiW}_{12} \mathrm{O}_{40}\right] \cdot 14 \mathrm{H}_{2} \mathrm{O} \cdot 2 \mathrm{ClCH}_{2} \mathrm{COOH}(2)$ (left); TGA/Mass plot of $\left[\mathrm{Cr}_{3}\left(\mu_{3}-\mathrm{O}\right)\left(\mathrm{ClCH}_{2} \mathrm{COO}\right)_{6}\left(\mathrm{H}_{2} \mathrm{O}\right)_{3}\right]_{4}\left[\mathrm{SiW}_{12} \mathrm{O}_{40}\right] \cdot 8 \mathrm{H}_{2} \mathrm{O} \cdot 2 \mathrm{ClCH}_{2} \mathrm{COOH}\left(\mathbf{2}^{\prime}\right)$ (right).

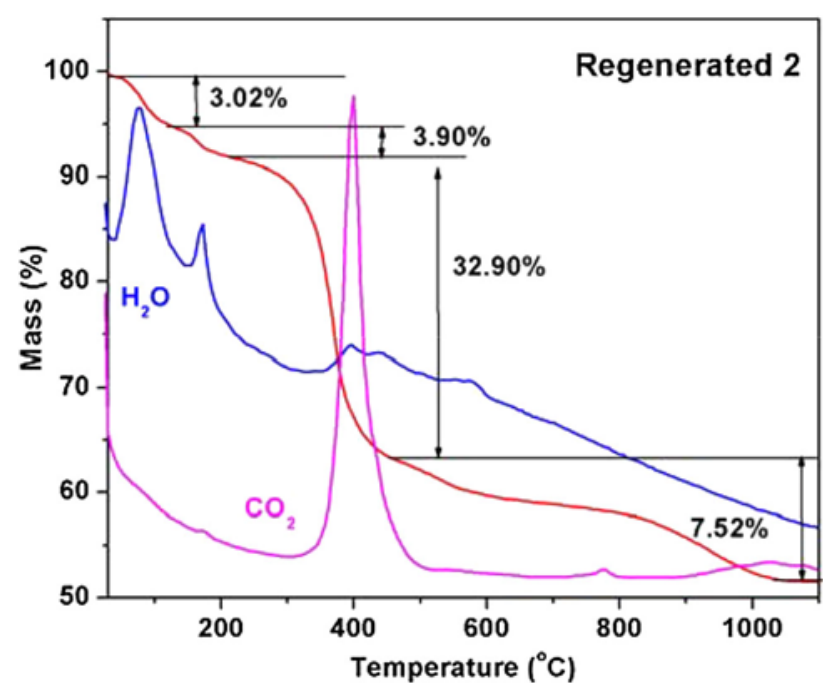

Figure 6. TGA/Mass plot of water regenerated compound (regenerated 2).

are isolated as crystalline materials with appropriate cations. ${ }^{26}$ The interest in this class of polyoxometalate based 'ionic crystals' has opened up a new field of research and enormous work has been done by Mizuno and co-workers, especially in terms of their applications in selective sorption and desorption of small molecules. ${ }^{17-25}$ They found that the systems showing this selective host-guest chemistry have the maximum macrocation/polyoxometalate ratio of $2: 1$, where the remaining negative charges are generally counterbalanced by alkali metal ions. In this contribution, we have discussed a series of ionic crystals consisting of macrocation/polyoxoanion ratio of $4: 1$ without the presence of alkali metal cations. We have described here the synthesis and structural characterization of the compound $\left[\mathrm{Fe}_{3}\left(\mu_{3}-\mathrm{O}\right)\left(\mathrm{ClCH}_{2} \mathrm{COO}\right)_{6}\left(\mathrm{H}_{2} \mathrm{O}\right)_{3}\right]_{4}\left[\mathrm{SiW}_{12} \mathrm{O}_{40}\right] \cdot 18 \mathrm{H}_{2} \mathrm{O}$. $2 \mathrm{ClCH}_{2} \mathrm{COOH}$ (1) (ratio of macrocation/polyoxoanions is $4: 1$ ). Compound $\mathbf{1}$, on heating at $85^{\circ} \mathrm{C}$ for 3.5 hours in an open oil bath, undergoes single crystal to single crystal transformation resulting in a dehydrated compound $\left[\mathrm{Fe}_{3}\left(\mu_{3}-\mathrm{O}\right)\left(\mathrm{ClCH}_{2} \mathrm{COO}\right)_{6}\left(\mathrm{H}_{2} \mathrm{O}\right)_{3}\right]_{4}\left[\mathrm{SiW}_{12} \mathrm{O}_{40}\right] \cdot 10 \mathrm{H}_{2} \mathrm{O}$. $2 \mathrm{ClCH}_{2} \mathrm{COOH}\left(\mathbf{1}^{\prime}\right)$. When the crystals of compound $\mathbf{1}$ are heated at $135^{\circ} \mathrm{C}$ for 3.5 hours in an open oil bath, relatively more dehydrated compound $\left[\mathrm{Fe}_{3}\left(\mu_{3}-\right.\right.$ 

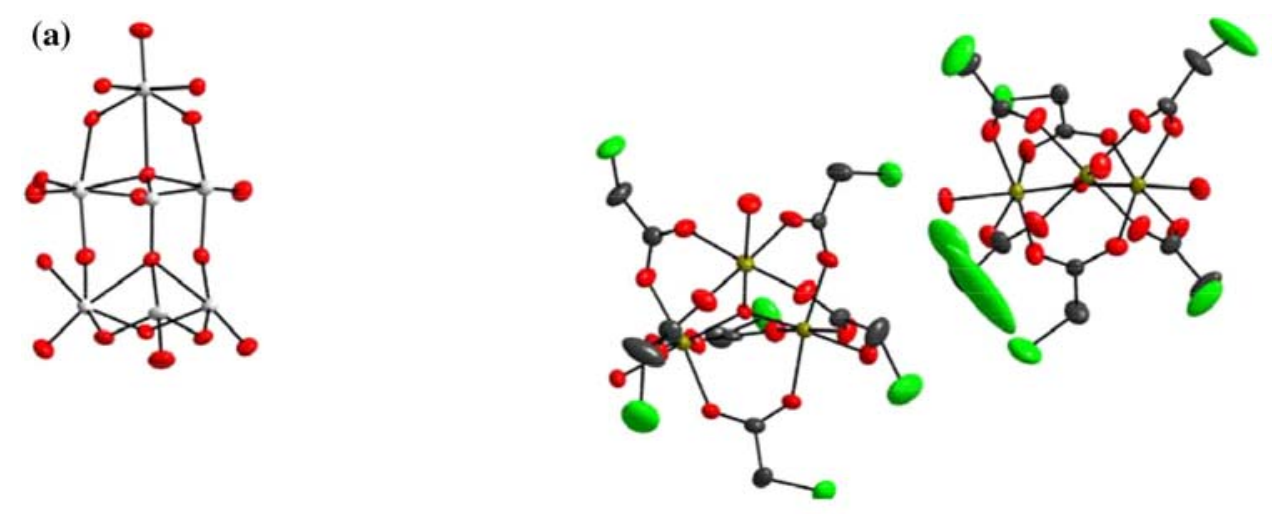

(b)

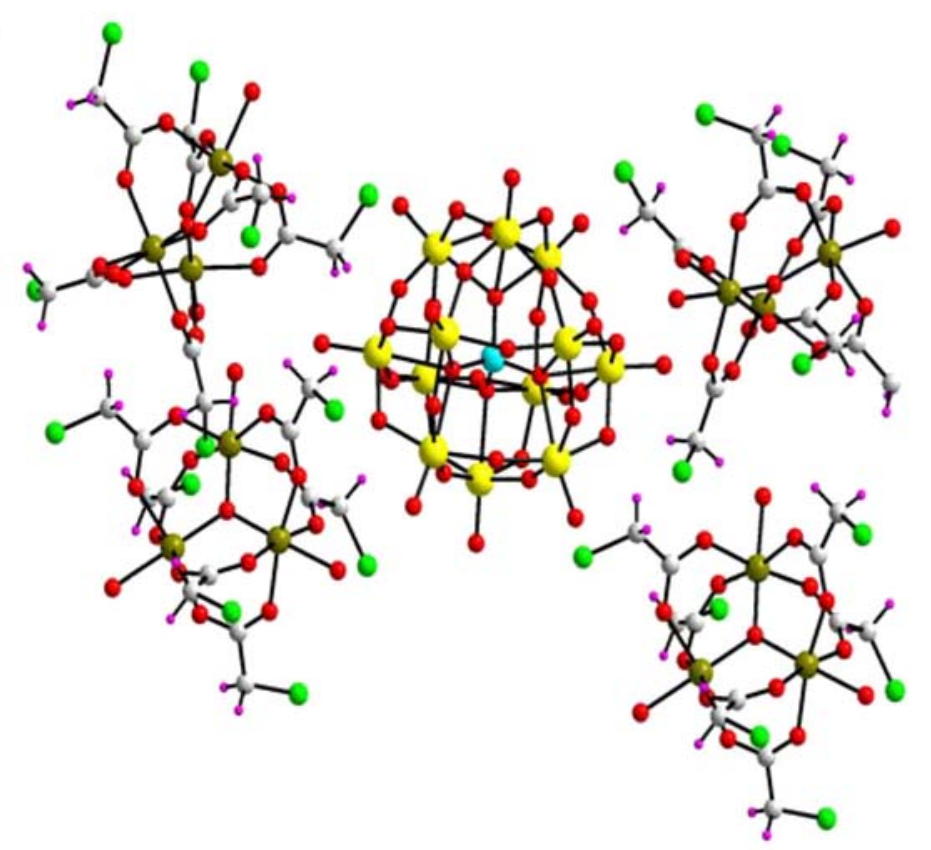

Figure 7. (a) Thermal ellipsoidal representation (50\% probability level) of the asymmetric unit of $\left[\mathrm{Fe}_{3}\left(\mu_{3}-\mathrm{O}\right)\left(\mathrm{ClCH}_{2} \mathrm{COO}\right)_{6}\left(\mathrm{H}_{2} \mathrm{O}\right)_{3}\right]_{4}\left[\mathrm{SiW}_{12} \mathrm{O}_{40}\right] \cdot 18 \mathrm{H}_{2} \mathrm{O} \cdot 2 \mathrm{ClCH}_{2} \mathrm{COOH}(\mathbf{1})$ excluding lattice solvent water and chloroacetic acid and hydrogen atoms. (b) The molecular structure of compound 1, excluding lattice water and chloroacetic acid molecules. Color code: Mo, yellow; $\mathrm{Si}$, cyan; $\mathrm{O}$, red; $\mathrm{Fe}$, dark green; $\mathrm{C}$, gray; $\mathrm{Cl}$, light green; $\mathrm{H}$, purple.

$\left.\mathrm{O})\left(\mathrm{ClCH}_{2} \mathrm{COO}\right)_{6}\left(\mathrm{H}_{2} \mathrm{O}\right)_{3}\right]_{4}\left[\mathrm{SiW}_{12} \mathrm{O}_{40}\right] \cdot 8 \mathrm{H}_{2} \mathrm{O} \cdot 2 \mathrm{ClCH}_{2}$ $\mathrm{COOH}\left(\mathbf{1}^{\prime \prime}\right)$ is obtained. Both these dehydrated compounds $\left(\mathbf{1}^{\prime}\right.$ and $\left.\mathbf{1}^{\prime \prime}\right)$ have been characterized by single crystal X-ray structure determination. One of these dehydrated compounds, $\mathbf{1}^{\prime}$ on exposing to water vapor for one week regenerates to compound $\mathbf{1}$ (regenerated 1). The single crystals of regenerated 1 exhibited similar cell parameters as those of parent $\mathbf{1}$ (the single crystal structure of regenerated 1 could not be determined due to its poor data quality). The crystals of regenerated 1 were further characterized by routine spectroscopic analysis, TGA-Mass studies and powder X-ray diffraction studies. We have shown similar crystal to crystal transformations mediated by chromium analogue compounds as shown below (unlike compound $\mathbf{1}$, chromium analogue 2 resulted in the same dehydrated compound, $\mathbf{2}^{\prime}$, even when 2 was heated at $85^{\circ} \mathrm{C}$ as well as $135^{\circ} \mathrm{C}$ ). That is,

$$
\begin{aligned}
& {\left[\mathrm{Cr}_{3}\left(\mu_{3}-\mathrm{O}\right)\left(\mathrm{ClCH}_{2} \mathrm{COO}\right)_{6}\left(\mathrm{H}_{2} \mathrm{O}\right)_{3}\right]_{4}\left[\mathrm{SiW}_{12} \mathrm{O}_{40}\right] \cdot} \\
& \quad 14 \mathrm{H}_{2} \mathrm{O} \cdot 2 \mathrm{ClCH}_{2} \mathrm{COOH}(2) \\
& (\mathbf{2})+\text { heated at } 85^{\circ} \mathrm{C} / 135^{\circ} \mathrm{C} \\
& \quad \rightarrow\left[\mathrm{Cr}_{3}\left(\mu_{3}-\mathrm{O}\right)\left(\mathrm{ClCH}_{2} \mathrm{COO}\right)_{6}\left(\mathrm{H}_{2} \mathrm{O}\right)_{3}\right]_{4}\left[\mathrm{SiW}_{12} \mathrm{O}_{40}\right] \cdot \\
& 8 \mathrm{H}_{2} \mathrm{O} \cdot 2 \mathrm{ClCH}_{2} \mathrm{COOH}\left(\mathbf{2}^{\prime}\right)
\end{aligned}
$$$$
\mathbf{2}^{\prime}+\text { exposure to water vapor } \rightarrow \text { regenerated } 2
$$

We have thus demonstrated here a reversible crystal to crystal transformation mediated by compounds $\mathbf{1}$ and 2 through exclusion and inclusion of water molecules in 
(a)
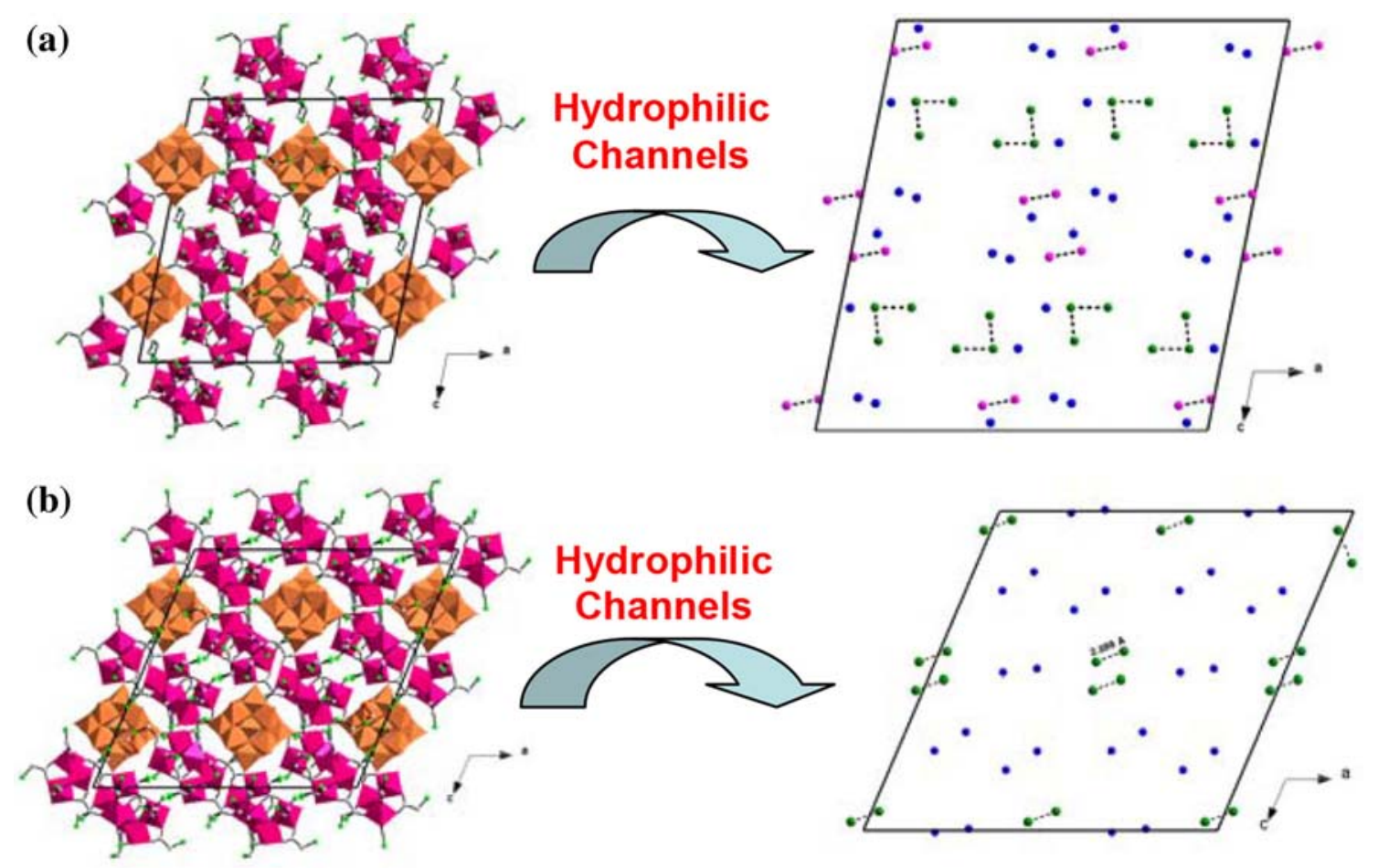

Figure 8. (a) The unit cell packing diagram of $\left[\mathrm{Fe}_{3}\left(\mu_{3}-\mathrm{O}\right)\left(\mathrm{ClCH}_{2} \mathrm{COO}\right)_{6}\left(\mathrm{H}_{2} \mathrm{O}\right)_{3}\right]_{4}\left[\mathrm{SiW}_{12} \mathrm{O}_{40}\right] \cdot 18 \mathrm{H}_{2} \mathrm{O} \cdot 2$ $\mathrm{ClCH}_{2} \mathrm{COOH}$ (1) which shows the arrangement of lattice water molecules in hydrophilic channels; (b) The unit cell packing diagram of $\left[\mathrm{Fe}_{3}\left(\mu_{3}-\mathrm{O}\right)\left(\mathrm{ClCH}_{2} \mathrm{COO}\right)_{6}\left(\mathrm{H}_{2} \mathrm{O}\right)_{3}\right]_{4}\left[\mathrm{SiW}_{12} \mathrm{O}_{40}\right] \cdot 10 \mathrm{H}_{2} \mathrm{O} \cdot 2 \mathrm{ClCH}_{2} \mathrm{COOH}$ (dehydrated $\mathbf{1 - 8 5}^{\circ}$ ) which shows the arrangement of lattice water molecules in hydrophilic channels.

respective ionic crystals. The parent compounds $\mathbf{1}$ and $\mathbf{2}$, and their dehydrated forms, $\mathbf{1}^{\prime}, \mathbf{1}^{\prime \prime}$ and $\mathbf{2}^{\prime}$ are unambiguously characterized by single crystal X-ray crystallography. The regenerated $\mathbf{1}$ and $\mathbf{2}$ are characterized by determining their unit cell parameters and comparing these unit cell parameters with those of the parent compounds $\mathbf{1}$ and $\mathbf{2}$. All these compounds are additionally characterized by IR and UV-visible spectra, powder Xray diffraction (PXRD) studies and their TGA analyses.

\section{Experimental}

\subsection{Materials}

All the chemicals were reagent grade and used without any further purification. The distilled water was used throughout the work. $\mathrm{H}_{4} \mathrm{SiW}_{12} \mathrm{O}_{40}$, hydrochloric acid and anhydrous $\mathrm{FeCl}_{3}$ were acquired from Merck. $\mathrm{ClCH}_{2} \mathrm{COOH}$ was received from S. D. Fine Chemicals. $\mathrm{Cr}\left(\mathrm{NO}_{3}\right)_{3} \cdot 9 \mathrm{H}_{2} \mathrm{O}$ was obtained from Loba Chemicals.

\subsection{Physical measurements}

Infrared spectra of solid samples were obtained as $\mathrm{KBr}$ pellets on a JASCO -5300 FT-IR spectrophotometer. A Cary 100 Bio $\mathrm{UV} /$ vis spectrophotometer was used to record the electronic absorption spectra. Thermogravimetric analyses were carried out on a STA 409 PC analyzer and corresponding masses were analyzed by QMS $403 \mathrm{C}$ mass analyzer, under the flow of $\mathrm{N}_{2}$ gas with a heating rate of $5^{\circ} \mathrm{C} \mathrm{min}^{-1}$, in the temperature range of $30-1100^{\circ} \mathrm{C}$. The Powder X-ray diffraction patterns were recorded using $\mathrm{Cu}-\mathrm{K} \alpha(\lambda=1.54 \AA)$ radiation on a Phillips PW 3710 diffractometer at a scanning speed of $3^{\circ} \mathrm{min}^{-1}$.

\subsection{Synthesis and characterization}

$2.3 \mathrm{a} \quad\left[\mathrm{Fe}_{3}\left(\mu_{3}-\mathrm{O}\right)\left(\mathrm{ClCH}_{2} \mathrm{COO}\right)_{6}\left(\mathrm{H}_{2} \mathrm{O}\right)_{3}\right]_{4}$ $\left[\mathrm{Si} \mathrm{W}_{12} \mathrm{O}_{40}\right] .18 \mathrm{H}_{2} \mathrm{O} .2 \mathrm{ClCH}_{2} \mathrm{COOH}(\mathbf{1})$ : To a $100 \mathrm{~mL}$ of water, $\mathrm{H}_{4} \mathrm{SiW}_{12} \mathrm{O}_{40}(0.5 \mathrm{~g}, 0.17 \mathrm{mmol}), 5 \mathrm{~mL}$ of $2.3 \mathrm{M}$ $\mathrm{HCl}$ were added, followed by the addition of $\mathrm{ClCH}_{2} \mathrm{COOH}$ $(5.5 \mathrm{~g}, 58.20 \mathrm{mmol})$. The $\mathrm{pH}$ of the resulting reaction mixture was adjusted to 2.0 by $5 \mathrm{M} \mathrm{NaOH}$ solution. To this solution, $\mathrm{FeCl}_{3}(0.34 \mathrm{~g}, 2.10 \mathrm{mmol})$ was added and the resulting solution was stirred for an hour, then filtered and the filtrate was kept open at room temperature. After one week, settled red colored crystals were collected, dried at room temperature and used for further studies. Yield: $0.45 \mathrm{~g}$ (39\% based on W). IR (KBr pellet): $\left(v / \mathrm{cm}^{-1}\right)$ : 3342, 2945, 1734, 1614 (vs, $\left.v_{\text {asym }}(\mathrm{OCO})\right), 1431,1392$ (vs, $\left.v_{\text {sym }}(\mathrm{OCO})\right), 1259,1016,974$ $\left(\mathrm{s}, v_{\text {asym }}(\mathrm{W}=\mathrm{O})\right), 920\left(\mathrm{br}, v_{\text {asym }}(\mathrm{Si}-\mathrm{O})\right), 798$ (br, $v_{\text {asym }}(\mathrm{W}-$ Oc-W)), 692 (m, $\left.v_{\text {asym }}\left(\mathrm{Fe}_{3}-\mathrm{O}\right)\right)$, 574, 507. Anal. calcd. for $\mathrm{C}_{52} \mathrm{H}_{114} \mathrm{Cl}_{26} \mathrm{Fe}_{12} \mathrm{O}_{126} \mathrm{SiW}_{12}: \mathrm{C}, 9.49 ; \mathrm{H}, 1.75 \%$. Found: $\mathrm{C}$, 9.88; $\mathrm{H}, 2.01 \%$. 


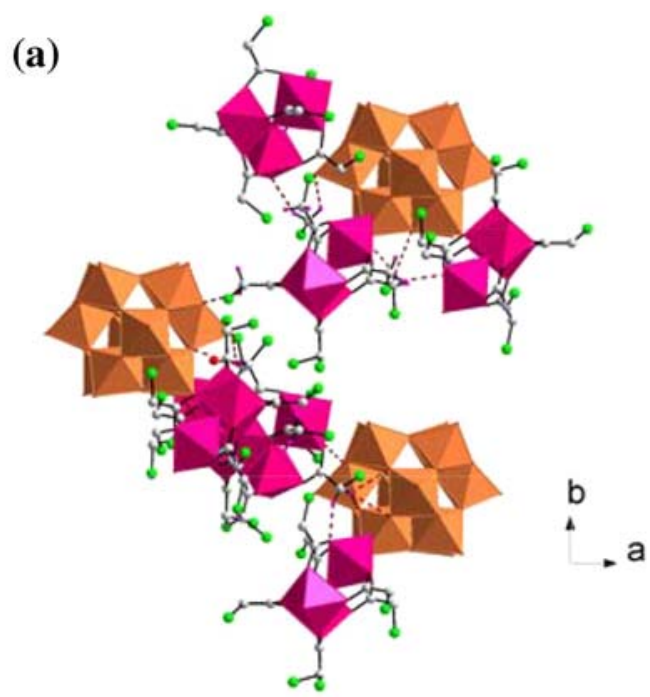

(b)

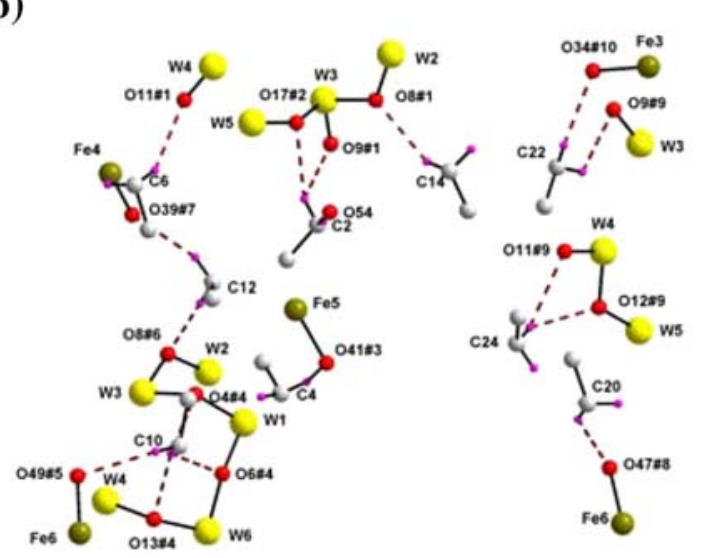

(c)

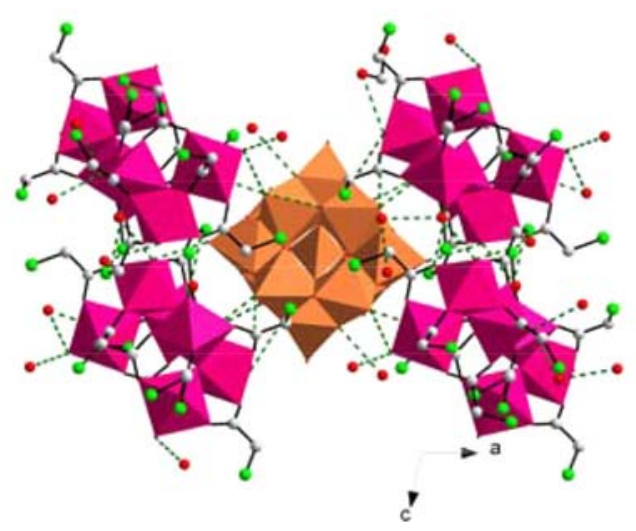

(d)

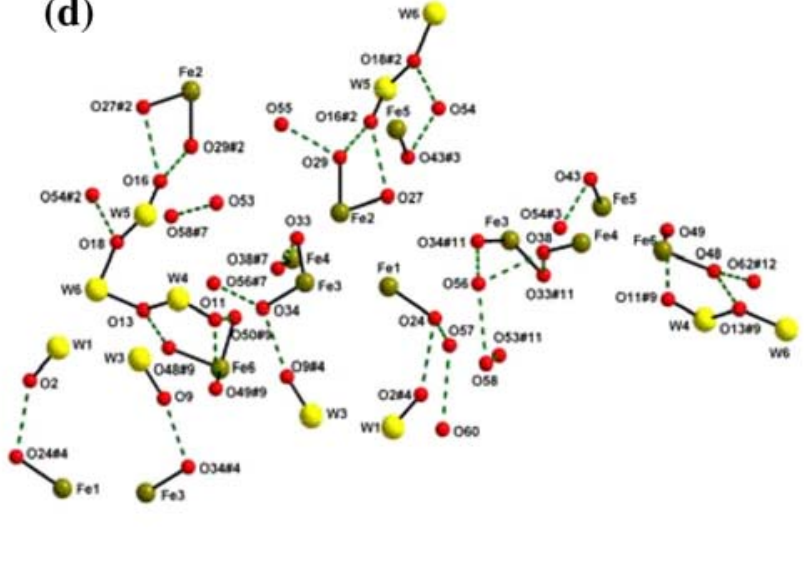

Figure 9. (a) $\mathrm{C}-\mathrm{H} \cdots \mathrm{O}$ hydrogen bonding interactions in $\left[\mathrm{Fe}_{3}\left(\mu_{3}-\mathrm{O}\right)\left(\mathrm{ClCH}_{2} \mathrm{COO}\right)_{6}\left(\mathrm{H}_{2} \mathrm{O}\right)_{3}\right]_{4}\left[\mathrm{SiW}_{12} \mathrm{O}_{40}\right]$. $18 \mathrm{H}_{2} \mathrm{O} \cdot 2 \mathrm{ClCH}_{2} \mathrm{COOH}(\mathbf{1})$. (b) $\mathrm{C}-\mathrm{H} \cdot \cdots \mathrm{O}$ hydrogen bonding interactions with relevant symmetry codes in compound 1. (c) $\mathrm{O}-\mathrm{H} \cdots \mathrm{O}$ hydrogen bonding interactions in compound 1. (d) $\mathrm{O}-\mathrm{H} \cdots \mathrm{O}$ hydrogen bonding interactions with relevant symmetry codes in compound $\mathbf{1}$. Color code: $\mathrm{W}$, yellow; $\mathrm{O}$, red; Fe, dark green; $\mathrm{Cl}$, light green; $\mathrm{C}$, gray; $\mathrm{H}$, purple; $\left[\mathrm{Fe}_{3}\left(\mu_{3}-\mathrm{O}\right)\left(\mathrm{ClCH}_{2} \mathrm{COO}\right)_{6}\left(\mathrm{H}_{2} \mathrm{O}\right)_{3}\right]^{1+}$ cluster, purple polyhedra; $\left[\mathrm{SiW}_{12} \mathrm{O}_{40}\right]^{4-}$ cluster, golden yellow polyhedra; $\mathrm{C}-\mathrm{H}$. . O hydrogen bonding interactions, brown dotted lines; $\mathrm{O}-\mathrm{H} \cdots \mathrm{O}$ hydrogen bonding interactions green dotted lines.

$2.3 \mathrm{~b}\left[\mathrm{Fe}_{3}\left(\mu_{3}-\mathrm{O}\right)\left(\mathrm{ClC}_{2} \mathrm{COO}\right)_{6}\left(\mathrm{H}_{2} \mathrm{O}\right)_{3}\right]_{4}$

$\left[\mathrm{Si} \mathrm{W}_{12} \mathrm{O}_{40}\right] \cdot 10 \mathrm{H}_{2} \mathrm{O} \cdot 2 \mathrm{ClC}_{2} \mathrm{COOH}\left(\mathbf{1}^{\prime}\right)$ : Compound $\mathbf{1}$, on heating in the temperature range of $80-85^{\circ} \mathrm{C}$ for $3.5 \mathrm{~h}$, produced $\mathbf{1}^{\prime}$. IR (KBr pellet): $\left(\nu / \mathrm{cm}^{-1}\right)$ : 3296, 2945, 1728, 1610 (vs, $\left.v_{\text {asym }}(\mathrm{OCO})\right), 1431,1394$ (vs, $\left.v_{\text {sym }}(\mathrm{OCO})\right), 1259,1016$, $974\left(\mathrm{~s}, \nu_{\text {asym }}(\mathrm{W}=\mathrm{O})\right), 918\left(\mathrm{br}, \nu_{\text {asym }}(\mathrm{Si}-\mathrm{O})\right), 798\left(\mathrm{br}, \nu_{\text {asym }}(\mathrm{W}-\right.$ Oc-W)), $690\left(\mathrm{~m}, \nu_{\text {asym }}\left(\mathrm{Fe}_{3}-\mathrm{O}\right)\right), 578,509$. Anal. calcd. for $\mathrm{C}_{52} \mathrm{H}_{98} \mathrm{Cl}_{26} \mathrm{Fe}_{12}$

$\mathrm{O}_{118} \mathrm{SiW}_{12}: \mathrm{C}, 9.70 ; \mathrm{H}, 1.53 \%$. Found: C, 10.11; H, $1.49 \%$.

$2.3 \mathrm{c} \quad\left[\mathrm{Fe}_{3}\left(\mu_{3}-\mathrm{O}\right)\left(\mathrm{ClCH}_{2} \mathrm{COO}\right)_{6}\left(\mathrm{H}_{2} \mathrm{O}\right)_{3}\right]_{4}$

[ $\left.\mathrm{Si}_{12} \mathrm{O}_{40}\right] \cdot 8 \mathrm{H}_{2} \mathrm{O} \cdot 2 \mathrm{ClCH}_{2} \mathrm{COOH}\left(\mathbf{1}^{\prime \prime}\right)$ : Compound 1 , on heating in the temperature range of $130-140^{\circ} \mathrm{C}$ for $3.5 \mathrm{~h}$, produced 1". IR (KBr pellet): $\left(\mathrm{v} / \mathrm{cm}^{-1}\right): 3306,2949$, 2334, 1738, 1614 (vs, $v_{\text {asym }}(\mathrm{OCO})$ ), 1550, 1429, 1398 (vs, $\left.v_{\text {sym }}(\mathrm{OCO})\right), 1259,1016,974\left(\mathrm{~s}, v_{\text {asym }}(\mathrm{W}=\mathrm{O})\right), 918$ (br, $\left.\nu_{\text {asym }}(\mathrm{Si}-\mathrm{O})\right), 800$ (br, $\left.\nu_{\text {asym }}(\mathrm{W}-\mathrm{Oc}-\mathrm{W})\right), 690\left(\mathrm{~m}, \mathrm{v}_{\text {asym }}\left(\mathrm{Fe}_{3}-\right.\right.$ O)), 578, 511. Anal. calcd. for $\mathrm{C}_{52} \mathrm{H}_{94} \mathrm{Cl}_{26} \mathrm{Fe}_{12} \mathrm{O}_{116}$ $\mathrm{SiW}_{12}$ :C, 9.76; H, 1.48\%. Found: C, 9.59; H, $1.55 \%$.

$2.3 \mathrm{~d}\left[\mathrm{Fe}_{3}\left(\mu_{3}-\mathrm{O}\right)\left(\mathrm{ClC} \mathrm{H}_{2} \mathrm{COO}\right)_{6}\left(\mathrm{H}_{2} \mathrm{O}\right)_{3}\right]_{4}$ $\left[\mathrm{Si} \mathrm{W}_{12} \mathrm{O}_{40}\right] \cdot 18 \mathrm{H}_{2} \mathrm{O} \cdot 2 \mathrm{ClC} \mathrm{H}_{2} \mathrm{COOH}($ regenerated 1$)$ : $\mathbf{1}^{\prime}$, on exposure to water vapor for the period of one week produced regenerated 1 , and was characterized by IR, PXRD and TGA/Mass analysis and by comparing unit cell parameters of the regenerated one with those of parent $\mathbf{1}$.

2.3e $\left[\mathrm{Cr}_{3}\left(\mu_{3}-\mathrm{O}\right)\left(\mathrm{ClC}_{2} \mathrm{COO}\right)_{6}\left(\mathrm{H}_{2} \mathrm{O}\right)_{3}\right]_{4}$ [ $\left.\mathrm{Si} \mathrm{W}_{12} \mathrm{O}_{40}\right] \cdot 14 \mathrm{H}_{2} \mathrm{O} \cdot 2 \mathrm{ClC} \mathrm{H}_{2} \mathrm{COOH}(2)$ : To $100 \mathrm{~mL}$ of water, $\mathrm{H}_{4} \mathrm{SiW}_{12} \mathrm{O}_{40}(0.5 \mathrm{~g}, 0.17 \mathrm{mmol}), 5 \mathrm{~mL}$ of $2.3 \mathrm{M}$ $\mathrm{HCl}$ were added followed by the addition of $\mathrm{ClCH}_{2} \mathrm{COOH}$ $(5.5 \mathrm{~g}, 58.20 \mathrm{mmol})$. The $\mathrm{pH}$ of the resulting reaction mixture 
Table 2. Geometrical parameters of the C-H. . .O hydrogen bonds $\left(\AA,^{\circ}\right)$ involved in supramolecular network of compound $\left[\mathrm{Fe}_{3}\left(\mu_{3}-\mathrm{O}\right)\left(\mathrm{ClCH}_{2} \mathrm{COO}\right)_{6}\left(\mathrm{H}_{2} \mathrm{O}\right)_{3}\right]_{4}\left[\mathrm{SiW}_{12} \mathrm{O}_{40}\right] \cdot{ }_{18} \mathrm{H}_{2} \mathrm{O}$. $2 \mathrm{ClCH}_{2} \mathrm{COOH}(\mathbf{1})$, where $\mathrm{D}=$ donor; $\mathrm{A}=$ acceptor.

\begin{tabular}{|c|c|c|c|c|}
\hline $\mathrm{D}-\mathrm{H} \cdot \cdots \mathrm{A}$ & $\mathrm{d}(\mathrm{D} \cdots \mathrm{H})$ & $\mathrm{d}(\mathrm{H} \cdots \mathrm{A})$ & $\mathrm{d}(\mathrm{D} \cdots \mathrm{A})$ & $\angle$ (DHA \\
\hline C2-H2A....O54 & 0.97 & 2.59 & $3.558(19)$ & 174.6 \\
\hline C2-H2B....O9\#1 & 0.97 & 2.61 & $3.315(15)$ & 129.9 \\
\hline C2-H2B....O17\#2 & 0.97 & 2.56 & $3.349(14)$ & 138.7 \\
\hline C4-H4B....O41\#3 & 0.97 & 2.80 & $3.500(16)$ & 129.8 \\
\hline C6-H6А....O11\#1 & 0.97 & 2.63 & $3.506(17)$ & 150.0 \\
\hline C10-H10A...O4\#4 & 0.97 & 3.02 & $3.569(15)$ & 117.2 \\
\hline C10-H10A....О6\#4 & 0.97 & 2.81 & $3.494(15)$ & 128.6 \\
\hline C10-H10A....O13\#4 & 0.97 & 2.62 & $3.428(14)$ & 141.4 \\
\hline C10-H10A....O49\#5 & 0.97 & 2.60 & $3.412(13)$ & 141.0 \\
\hline C12-H12B....O8\#6 & 0.97 & 2.40 & $3.368(13)$ & 178.4 \\
\hline C12-H12A....O39\#7 & 0.97 & 2.67 & $3.541(14)$ & 150.2 \\
\hline C14-H14A...O8\#1 & 0.97 & 2.56 & $3.366(13)$ & 140.6 \\
\hline С20-H20A....О47\#8 & 0.97 & 2.59 & $3.378(15)$ & 138.2 \\
\hline C22-H22A....O9\#9 & 0.97 & 2.88 & $3.471(12)$ & 120.0 \\
\hline C22-H22B...O34\#10 & 0.97 & 2.65 & $3.272(12)$ & 122.6 \\
\hline C24-H24B...O11\#9 & 0.97 & 2.71 & $3.318(16)$ & 121.3 \\
\hline C24-H24B...O12\#9 & 0.97 & 2.67 & $3.530(15)$ & 148.3 \\
\hline
\end{tabular}

Symmetric transformations used to generate equivalent atoms: \#1 x, 1-y, 0.5+z; \#2 -x, 1-y, 1-z; \#3 0.5-x, 1.5-y, 2-z; \#4 0.5-x, 0.5-y, 1-z; \#5 x, -1+y, z; \#6 0.5-x, 0.5-y, 1-z; \#7 0.5-x, -0.5+y, 1.5-z; \#8 1-x, 2-y, 2-z; \#9 0.5-x, 1.5-y, 1-z; \#10 x, 1+y, z; \#11 0.5-x, 0.5+y, 1.5-z; \#12 x, 1+y, $1+\mathrm{z}$.

Table 3. Geometrical parameters of the O-H. . O hydrogen bonds $(\AA)$ involved in supramolecular network of compound $\left[\mathrm{Fe}_{3}\left(\mu_{3}-\mathrm{O}\right)\left(\mathrm{ClCH}_{2} \mathrm{COO}\right)_{6}\left(\mathrm{H}_{2} \mathrm{O}\right)_{3}\right]_{4}\left[\mathrm{SiW}_{12} \mathrm{O}_{40}\right]$. $18 \mathrm{H}_{2} \mathrm{O} \cdot 2 \mathrm{ClCH}_{2} \mathrm{COOH}(\mathbf{1})$.

\begin{tabular}{lccc}
\hline $\mathrm{O}(16)-\mathrm{O}(27) \# 2$ & $3.019(10)$ & $\mathrm{O}(16)-\mathrm{O}(29) \# 2$ & $3.039(11)$ \\
$\mathrm{O}(18)-\mathrm{O}(54) \# 2$ & $3.071(14)$ & $\mathrm{O}(13)-\mathrm{O}(48) \# 9$ & $2.898(10)$ \\
$\mathrm{O}(11)-\mathrm{O}(49) \# 9$ & $2.906(10)$ & $\mathrm{O}(11)-\mathrm{O}(50) \# 9$ & $2.909(10)$ \\
$\mathrm{O}(9)-\mathrm{O}(34) \# 4$ & $2.723(9)$ & $\mathrm{O}(2)-\mathrm{O}(24) \# 4$ & $2.909(10)$ \\
$\mathrm{O}(24)-\mathrm{O}(57)$ & $2.636(18)$ & $\mathrm{O}(24)-\mathrm{O}(2) \# 4$ & $2.909(10)$ \\
$\mathrm{O}(34)-\mathrm{O}(9) \# 4$ & $2.723(9)$ & $\mathrm{O}(34)-\mathrm{O}(56) \# 7$ & $2.738(11)$ \\
$\mathrm{O}(29)-\mathrm{O}(16) \# 2$ & $3.039(11)$ & $\mathrm{O}(29)-\mathrm{O}(55)$ & $2.686(18)$ \\
$\mathrm{O}(33)-\mathrm{O}(38) \# 7$ & $2.867(10)$ & $\mathrm{O}(27)-\mathrm{O}(16) \# 2$ & $3.019(10)$ \\
$\mathrm{O}(38)-\mathrm{O}(56)$ & $2.709(12)$ & $\mathrm{O}(38)-\mathrm{O}(33) \# 11$ & $2.867(10)$ \\
$\mathrm{O}(48)-\mathrm{O}(13) \# 9$ & $2.898(10)$ & $\mathrm{O}(48)-\mathrm{O}(62) \# 12$ & $3.000(9)$ \\
$\mathrm{O}(43)-\mathrm{O}(54) \# 3$ & $2.775(15)$ & $\mathrm{O}(49)-\mathrm{O}(11) \# 9$ & $2.909(10)$ \\
$\mathrm{O}(53)-\mathrm{O}(58) \# 7$ & $2.820(3)$ & $\mathrm{O}(54)-\mathrm{O}(18) \# 2$ & $3.071(14)$ \\
$\mathrm{O}(54)-\mathrm{O}(43) \# 3$ & $2.775(15)$ & $\mathrm{O}(55)-\mathrm{O}(29)$ & $2.686(18)$ \\
$\mathrm{O}(56)-\mathrm{O}(34) \# 11$ & $2.738(11)$ & $\mathrm{O}(56)-\mathrm{O}(38)$ & $2.709(12)$ \\
$\mathrm{O}(56)-\mathrm{O}(58)$ & $2.910(3)$ & $\mathrm{O}(57)-\mathrm{O}(24)$ & $2.636(18)$ \\
$\mathrm{O}(57)-\mathrm{O}(60)$ & $3.030(5)$ & $\mathrm{O}(58)-\mathrm{O}(53) \# 11$ & $2.820(3)$ \\
\hline
\end{tabular}

Symmetric codes are already specified in the footnote of Table 2.

was adjusted to 2.0 by $5 \mathrm{M} \mathrm{NaOH}$ solution. To this solution, $\mathrm{Cr}\left(\mathrm{NO}_{3}\right)_{3} \cdot 9 \mathrm{H}_{2} \mathrm{O}(1.0 \mathrm{~g}, 2.50 \mathrm{mmol})$ was added and the resulting solution was stirred for an hour, then filtered and the filtrate was kept open at room temperature. After one week, settled green colored crystals were filtered, dried in room temperature and used for further studies. Yield: $0.52 \mathrm{~g}(46 \%$ based on W). IR (KBr pellet): $\left(\nu / \mathrm{cm}^{-1}\right)$ : 3557, 3335, 2949,

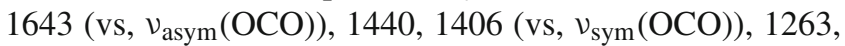



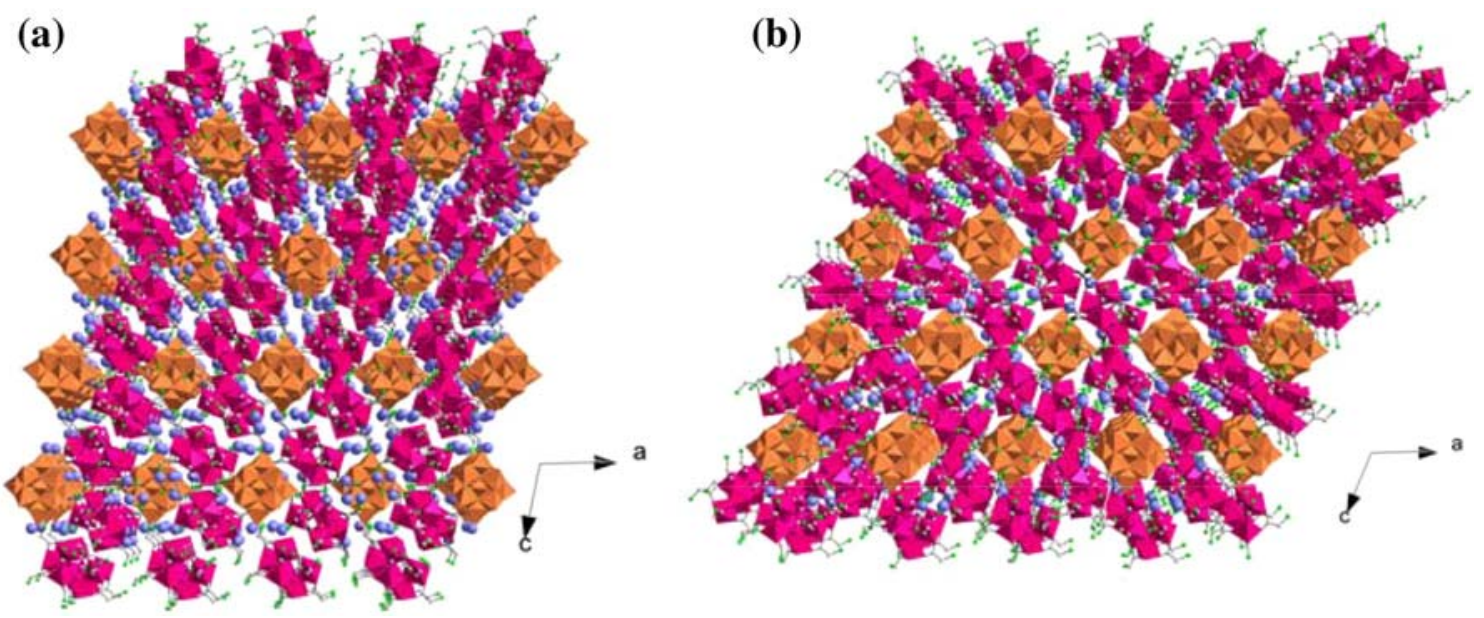

Figure 10. A perspective view of packing diagram along the crystallographic $b$ axis: (a) $\left[\mathrm{Fe}_{3}\left(\mu_{3}-\mathrm{O}\right)\left(\mathrm{ClCH}_{2} \mathrm{COO}\right)_{6}\left(\mathrm{H}_{2} \mathrm{O}\right)_{3}\right]_{4}\left[\mathrm{SiW}_{12} \mathrm{O}_{40}\right] \cdot 1_{2} \mathrm{H}_{2} \mathrm{O} \cdot 2 \mathrm{ClCH}_{2} \mathrm{COOH} \quad$ (1) and (b) $\left[\mathrm{Fe}_{3}\left(\mu_{3}-\mathrm{O}\right)\left(\mathrm{ClCH}_{2} \mathrm{COO}\right)_{6}\left(\mathrm{H}_{2} \mathrm{O}\right)_{3}\right]_{4}\left[\mathrm{SiW}_{12} \mathrm{O}_{40}\right] \cdot 10 \mathrm{H}_{2} \mathrm{O}$. $2 \mathrm{ClCH}_{2} \mathrm{COOH}\left(\mathbf{1}^{\prime}\right)$; Color code: $\left[\mathrm{Fe}_{3}\left(\mu_{3}-\mathrm{O}\right)\left(\mathrm{ClCH}_{2} \mathrm{COO}\right)_{6}\left(\mathrm{H}_{2} \mathrm{O}\right)_{3}\right]^{1+}$ cluster, purple polyhedra; $\left[\mathrm{SiW}_{12} \mathrm{O}_{40}\right]^{4-}$ cluster, golden yellow polyhedra; non-coordinated water molecules, blue; $\mathrm{Cl}$, light green.

Table 4. The inter nuclear distances between two $\left[\mathrm{SiW}_{12} \mathrm{O}_{40}\right]^{4-}$ cluster anions along crystallographic $c$ axis in compound $\mathbf{1}$ and $\mathbf{1}^{\prime}$.

$\left[\mathrm{Fe}_{3}\left(\mu_{3}-\mathrm{O}\right)\left(\mathrm{ClCH}_{2} \mathrm{COO}\right)_{6}\left(\mathrm{H}_{2} \mathrm{O}\right)_{3}\right]_{4} \quad\left[\mathrm{Fe}_{3}\left(\mu_{3}-\mathrm{O}\right)\left(\mathrm{ClCH}_{2} \mathrm{COO}\right)_{6}\left(\mathrm{H}_{2} \mathrm{O}\right)_{3}\right]_{4}$ $\left[\mathrm{SiW}_{12} \mathrm{O}_{40}\right] \cdot 18 \mathrm{H}_{2} \mathrm{O} \cdot 2 \mathrm{ClCH}_{2} \mathrm{COOH} \quad\left[\mathrm{SiW}_{12} \mathrm{O}_{40}\right] \cdot 10 \mathrm{H}_{2} \mathrm{O} \cdot 2 \mathrm{ClCH}_{2} \mathrm{COOH}$ (1) Internulear distance $(\AA)$ between $\left(\mathbf{1}^{\prime}\right)$ Internulear distance $(\AA)$ between two $\left[\mathrm{SiW}_{12} \mathrm{O}_{40}\right]^{4-}$ cluster anions two $\left[\mathrm{SiW}_{12} \mathrm{O}_{40}\right]^{4-}$ cluster anions

\begin{tabular}{llcr}
\hline $\mathrm{Si}-\mathrm{Si}$ & 17.78 & $\mathrm{Si}-\mathrm{Si}$ & 15.35 \\
$\mathrm{~W} 1-\mathrm{W} 1$ & 13.15 & W1A-W1A & 11.31 \\
W1 - W1 & 20.31 & W1A - W1B & 19.53 \\
W2 - W2 & 14.02 & W2A - W2A & 13.67 \\
W2 - W2 & 19.76 & W2A - W2B & 17.98 \\
W3 - W3 & 18.37 & W3 - W3 & 14.92 \\
W3 - W3 & 19.67 & W3 - W3 & 18.55 \\
W4 - W4 & 17.66 & W4A - W4A & 13.63 \\
W4 - W4 & 23.81 & W4B - W4A & 21.12 \\
W5 - W5 & 18.14 & W5A - W5B & 15.34 \\
W5 - W5 & 23.44 & W5A - W5B & 19.95 \\
W6 - W6 & 11.91 & W6A - W6A & 9.05 \\
W6-W6 & 24.43 & W6A - W6B & 22.18 \\
\hline
\end{tabular}

$974\left(\mathrm{~s}, \nu_{\text {asym }}(\mathrm{W}=\mathrm{O})\right), 920\left(\mathrm{br}, \nu_{\text {asym }}(\mathrm{Si}-\mathrm{O})\right), 800\left(\mathrm{br}, \nu_{\text {asym }}(\mathrm{W}-\right.$ Oc-W)), 706, 659 (m, $\left.v_{\text {asym }}\left(\mathrm{Cr}_{3}-\mathrm{O}\right)\right)$, 441. Anal. calcd. for $\mathrm{C}_{52} \mathrm{H}_{106} \mathrm{Cl}_{26} \mathrm{Cr}_{12} \mathrm{O}_{122} \mathrm{SiW}_{12}: \mathrm{C}, 9.66 ; \mathrm{H}, 1.65 \%$. Found: $\mathrm{C}$, 9.76; $\mathrm{H}, 1.58 \%$.

\section{3f $\left[\mathrm{Cr}_{3}\left(\mu_{3}-\mathrm{O}\right)\left(\mathrm{ClC} \mathrm{H}_{2} \mathrm{COO}\right)_{6}\left(\mathrm{H}_{2} \mathrm{O}\right)_{3}\right]_{4}$} $\left[\mathrm{Si} \mathrm{W}_{12} \mathrm{O}_{40}\right] \cdot 8 \mathrm{H}_{2} \mathrm{O} \cdot 2 \mathrm{ClC} \mathrm{H}_{2} \mathrm{COOH}\left(\mathbf{2}^{\prime}\right)$ : A bunch of crystalline compound $\mathbf{2}$, on heating in the temperature range of $135^{\circ} \mathrm{C}$ for $3.5 \mathrm{~h}$ or $85^{\circ} \mathrm{C}$ for $3.5 \mathrm{~h}$, produced same $\mathbf{2}^{\prime}$ compound. IR ( $\mathrm{KBr}$ pellet): $\left(\mathrm{v} / \mathrm{cm}^{-1}\right): 3557,3342,2953,1643$ (vs, $v_{\text {asym }}(\mathrm{OCO})$ ), 1440, 1410 (vs, $v_{\text {sym }}(\mathrm{OCO})$ ), 1261, 1016, $972\left(\mathrm{~s}, v_{\text {asym }}(\mathrm{W}=\mathrm{O})\right), 920\left(\mathrm{br}, \nu_{\text {asym }}(\mathrm{Si}-\mathrm{O})\right), 802\left(\mathrm{br}, \nu_{\text {asym }}(\mathrm{W}-\right.$ Oc-W)), 706, 655 (m, vasym $\left.\left(\mathrm{Cr}_{3}-\mathrm{O}\right)\right), 588$, 439. Anal. calcd. for $\mathrm{C}_{52} \mathrm{H}_{94} \mathrm{Cl}_{26} \mathrm{Cr}_{12} \mathrm{O}_{116} \mathrm{SiW}_{12}: \mathrm{C}, 9.82 ; \mathrm{H}, 1.49 \%$. Found: $\mathrm{C}, 10.06 ; \mathrm{H}, 1.55 \%$.

$2.3 \mathrm{~g}\left[\mathrm{Cr}_{3}\left(\mu_{3}-\mathrm{O}\right)\left(\mathrm{ClC} \mathrm{H} \mathrm{COO}_{6}\left(\mathrm{H}_{2} \mathrm{O}\right)_{3}\right]_{4}\right.$ $\left[\mathrm{Si} \mathrm{W}_{12} \mathrm{O}_{40}\right] \cdot 14 \mathrm{H}_{2} \mathrm{O} \cdot 2 \mathrm{ClC}_{2} \mathrm{COOH}($ regenerated 2$)$ : Compound $\mathbf{2}^{\prime}$, on exposure to water vapor for the period of one week, produced regenerated 2. It was characterized by IR, PXRD and TGA/Mass analysis. IR ( $\mathrm{KBr}$ pellet): $\left(v / \mathrm{cm}^{-1}\right): 3314,2951,1792,1643$ (vs, $v_{\text {asym }}($ OCO $\left.)\right), 1440$, 1406 (vs, $\left.v_{\text {sym }}(\mathrm{OCO})\right), 1263,1016,974$ (s, $\left.v_{\text {asym }}(\mathrm{W}=\mathrm{O})\right), 920$ (br, $\left.\nu_{\text {asym }}(\mathrm{Si}-\mathrm{O})\right), 800\left(\mathrm{br}, \nu_{\text {asym }}(\mathrm{W}-\mathrm{Oc}-\mathrm{W})\right), 706,655(\mathrm{~m}$, $\left.\nu_{\text {asym }}\left(\mathrm{Cr}_{3}-\mathrm{O}\right)\right), 588,532,428$. It was also characterized by comparing unit cell parameters of the regenerated one with those of parent 2. 


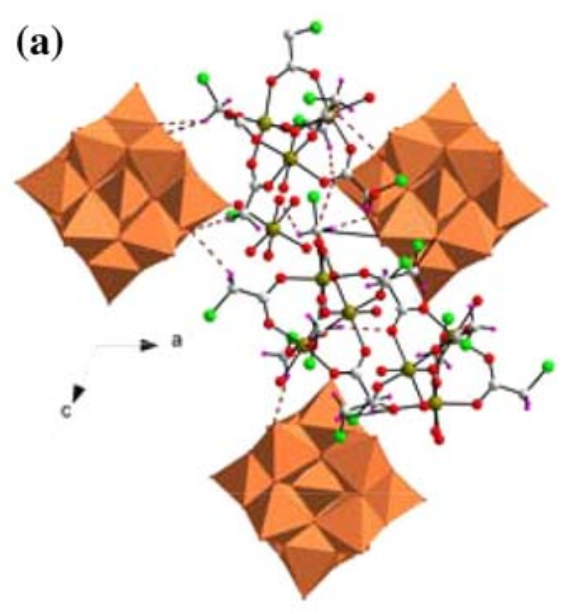

(c)

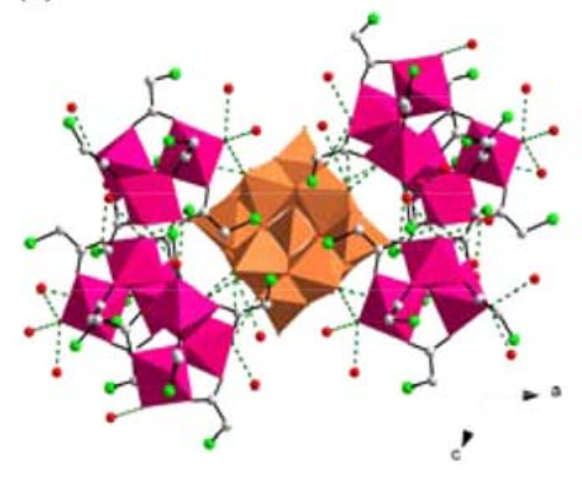

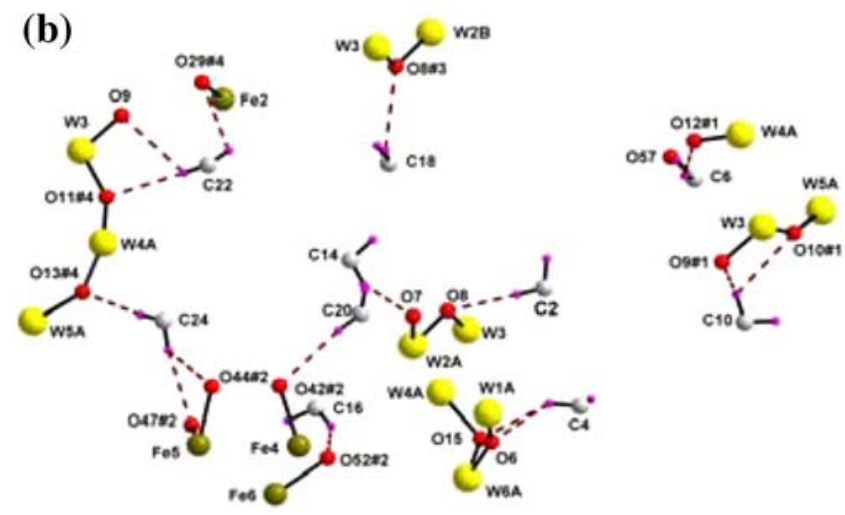

(d)

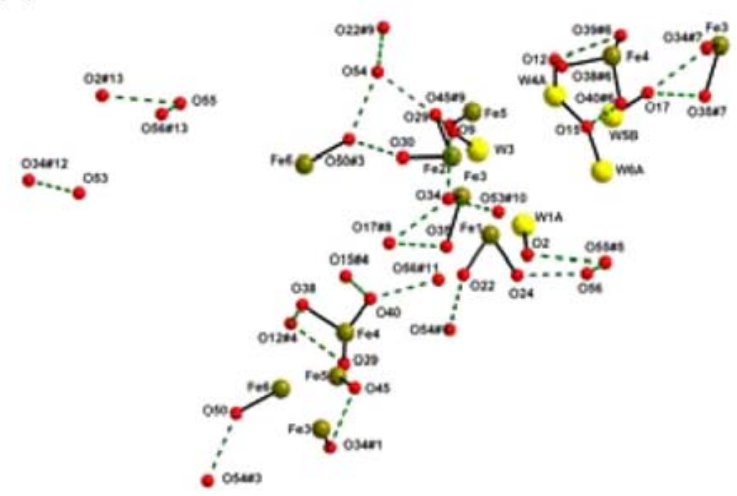

Figure 11. (a) The $\mathrm{C}-\mathrm{H} \cdots \mathrm{O}$ hydrogen bonding interactions in $\left[\mathrm{Fe}_{3}\left(\mu_{3}-\mathrm{O}\right)\left(\mathrm{ClCH}_{2} \mathrm{COO}\right)_{6}\left(\mathrm{H}_{2} \mathrm{O}\right)_{3}\right]_{4}$ $\left[\mathrm{SiW}_{12} \mathrm{O}_{40}\right] \cdot 10 \mathrm{H}_{2} \mathrm{O} \cdot 2 \mathrm{ClCH}_{2} \mathrm{COOH}\left(\mathbf{1}^{\prime}\right)$. (b) $\mathrm{C}-\mathrm{H} \cdots \mathrm{O}$ hydrogen bonding interactions with relevant symmetry codes in compound $\mathbf{1}^{\prime}$. (c) $\mathrm{O}-\mathrm{H} \cdots \mathrm{O}$ hydrogen bonding interactions in compound $\mathbf{1}^{\prime}$ ). (d) $\mathrm{O}-\mathrm{H} \cdots \mathrm{O}$ hydrogen bonding interactions with relevant symmetry codes in compound $\mathbf{1}^{\prime}$. Color code: same as in the caption of Figure 9.

\subsection{Single crystal structure determination}

Data were measured on a Bruker SMART APEX CCD area detector system $[\lambda($ Mo K $\alpha)=0.71073 \AA]$ with graphite monochromator. 2400 frames were recorded with an $\omega$ scan width of $0.3^{\circ}$, each for 10 second with a crystal detector distance of $60 \mathrm{~mm}$, and with a collimator of $0.5 \mathrm{~mm}$. The data were reduced using SAINTPLUS, ${ }^{27}$ the structures were solved using SHELXS-97, 28 and refined using SHELXL97. ${ }^{29}$ DIAMOND ${ }^{30}$ software was used for molecular graphics. All non-hydrogen atoms were refined anisotropically. Hydrogen atoms on chloroacetate moiety from the trinuclear cluster were introduced on calculated positions and included in the refinement riding on their respective parent atoms. We tried to locate the hydrogen atom of solvent water molecules through differential Fourier maps, but could not succeed. The lattice $\mathrm{ClCH}_{2} \mathrm{COOH}$ molecules were refined isotropically due to the considerable disorder. Summaries of the crystallographic data and structure determination parameters for compounds 1 , dehydrated $1-85^{\circ}\left(1^{\prime}\right)$ and dehydrated $1-135^{\circ}$ $\left(\mathbf{1}^{\prime \prime}\right)$ are presented in Table 1 . The crystal data and relevant parameters for compound $\mathbf{2}$, dehydrated $2-\mathbf{8 5}^{\circ} / \mathbf{1 3 5}^{\circ}\left(\mathbf{2}^{\prime}\right)$ are presented in Table 9.

\section{Results and Discussion}

\subsection{Electronic spectroscopy}

During crystal to crystal transformations, the metal centers do not change their coordination environments and hence the physical properties of the compounds $\mathbf{1}, \mathbf{1}^{\prime}, \mathbf{1}^{\prime \prime}$ and $\mathbf{2}, \mathbf{2}^{\prime}$ would not be expected to change. Therefore, UV-visible absorbance spectra have been studied by taking compounds $\mathbf{1}$ and $\mathbf{2}$ as representative compounds from each series. The shoulders which appeared at 420 $\mathrm{nm}$ and $460 \mathrm{~nm}$ (Figure 1, left) in the electronic absorption spectrum compound $\mathbf{1}$ are attributed to $\mathrm{O} \rightarrow \mathrm{Fe}^{3+}$ charge transfer, comparable to those found in other oxobridged Fe(III) compounds. ${ }^{31,32}$ The very weak features 
Table 5. Geometrical parameters of the $\mathrm{C}-\mathrm{H} \cdots \mathrm{O}$ hydrogen bonds $\left(\AA{ }^{\circ}\right)$ involved in supramolecular network of compound $\left[\mathrm{Fe}_{3}\left(\mu_{3}-\mathrm{O}\right)\right.$ $\left.\left(\mathrm{ClCH}_{2} \mathrm{COO}\right)_{6}\left(\mathrm{H}_{2} \mathrm{O}\right)_{3}\right]_{4}\left[\mathrm{SiW}_{12} \mathrm{O}_{40}\right] \cdot 10 \mathrm{H}_{2} \mathrm{O} \cdot 2 \mathrm{ClCH}_{2} \mathrm{COOH}\left(\mathbf{1}^{\prime}\right)$, where $\mathrm{D}=$ donor; $\mathrm{A}=$ acceptor.

\begin{tabular}{|c|c|c|c|c|}
\hline D-H $\cdots A$ & $\mathrm{~d}(\mathrm{D} \cdots \mathrm{H})$ & $\mathrm{d}(\mathrm{H} \cdots \mathrm{A})$ & $\mathrm{d}(\mathrm{D} \cdots \mathrm{A})$ & $\angle$ (DHA \\
\hline $\mathrm{C} 2-\mathrm{H} 2 \mathrm{~A} \ldots . . \mathrm{O} 8$ & 0.97 & 2.47 & $3.442(13)$ & 179.1 \\
\hline C4-H4B....O6 & 0.97 & 2.82 & $3.480(12)$ & 126.1 \\
\hline C4-H4B....O15 & 0.97 & 2.57 & $3.395(11)$ & 142.9 \\
\hline С6-H6А....O12\#1 & 0.97 & 2.58 & $3.444(15)$ & 148.0 \\
\hline C6-H6B....O57 & 0.97 & 2.71 & $3.410(3)$ & 129.9 \\
\hline C10-H10A...O9\#1 & 0.97 & 2.31 & $3.122(13)$ & 140.3 \\
\hline C10-H10A....O10\#1 & 0.97 & 2.75 & $3.387(12)$ & 123.6 \\
\hline C14-H14A....O7 & 0.97 & 2.53 & $3.401(15)$ & 150.2 \\
\hline C16-H16B....O52\#2 & 0.97 & 2.69 & $3.510(2)$ & 143.2 \\
\hline C18-H18A....O8\#3 & 0.97 & 2.83 & $3.554(12)$ & 131.8 \\
\hline C20-H20A....O42\#2 & 0.97 & 2.57 & $3.492(15)$ & 158.2 \\
\hline С22-H22A...O9\#4 & 0.97 & 2.95 & $3.588(12)$ & 124.3 \\
\hline C22-H22A....O11\#4 & 0.97 & 2.61 & $3.522(11)$ & 157.5 \\
\hline C22-H22B....О29\#4 & 0.97 & 2.79 & $3.364(11)$ & 119.0 \\
\hline C24-H24B...O13\#4 & 0.97 & 2.44 & $3.409(11) 173.9$ & \\
\hline C24-H24B...O44\#2 & 0.97 & 2.87 & $3.442(13)$ & 118.5 \\
\hline C24-H24A...O47\#2 & 0.97 & 2.55 & $3.509(13)$ & 170.2 \\
\hline
\end{tabular}

Symmetry transformations used to generate equivalent atoms: \#1 0.5-x, -0.5+y, 0.5-z; \#2 -x, -y, 1-z; \#3 0.5-x, 0.5-y, 1-z; \#4 x, 1-y, 0.5+z; \#5 $-1+\mathrm{x}, \mathrm{y}, \mathrm{z}$; \#6 x, 1-y, -0.5+z; \#7-0.5+x, 0.5+y, z; \#8 0.5+x, -0.5+y, z; \#9 $0.5-\mathrm{x}, 0.5+\mathrm{y}, 0.5-\mathrm{z}$; \#10 x, y, -1+z; \#11 -x, y, 0.5-z; \#12 x, y, 1+z; \#13 $1+\mathrm{x}, \mathrm{y}, \mathrm{z}$.

Table 6. Geometrical parameters of the $\mathrm{O}-\mathrm{H}$. . O O hydrogen bonds $(\AA)$ involved in supramolecular network of compound $\left[\mathrm{Fe}_{3}\left(\mu_{3}-\mathrm{O}\right)\left(\mathrm{ClCH}_{2} \mathrm{COO}\right)_{6}\left(\mathrm{H}_{2} \mathrm{O}\right)_{3}\right]_{4}\left[\mathrm{SiW}_{12} \mathrm{O}_{40}\right] \cdot 10 \mathrm{H}_{2} \mathrm{O}$. $2 \mathrm{ClCH}_{2} \mathrm{COOH}\left(\mathbf{1}^{\prime}\right)$.

\begin{tabular}{lccc}
\hline $\mathrm{O}(2)-\mathrm{O}(24)$ & $2.886(9)$ & $\mathrm{O}(2)-\mathrm{O}(55) \# 5$ & $3.098(13)$ \\
$\mathrm{O}(9)-\mathrm{O}(29)$ & $2.733(9)$ & $\mathrm{O}(12)-\mathrm{O}(38) \# 6$ & $2.913(9)$ \\
$\mathrm{O}(12)-\mathrm{O}(39) \# 6$ & $3.026(10)$ & $\mathrm{O}(15)-\mathrm{O}(40) \# 6$ & $2.753(8)$ \\
$\mathrm{O}(17)-\mathrm{O}(34) \# 7$ & $2.840(9)$ & $\mathrm{O}(17)-\mathrm{O}(35) \# 7$ & $3.052(9)$ \\
$\mathrm{O}(22)-\mathrm{O}(54) \# 1$ & $3.079(11)$ & $\mathrm{O}(24)-\mathrm{O}(56)$ & $2.738(10)$ \\
$\mathrm{O}(29)-\mathrm{O}(54)$ & $2.773(10)$ & $\mathrm{O}(30)-\mathrm{O}(50) \# 3$ & $2.878(9)$ \\
$\mathrm{O}(34)-\mathrm{O}(17) \# 8$ & $2.840(9)$ & $\mathrm{O}(34)-\mathrm{O}(45) \# 9$ & $3.061(10)$ \\
$\mathrm{O}(34)-\mathrm{O}(53) \# 10$ & $2.560(18)$ & $\mathrm{O}(35)-\mathrm{O}(17) \# 8$ & $3.052(9)$ \\
$\mathrm{O}(38)-\mathrm{O}(12) \# 4$ & $2.913(9)$ & $\mathrm{O}(39)-\mathrm{O}(12) \# 4$ & $3.026(10)$ \\
$\mathrm{O}(40)-\mathrm{O}(15) \# 4$ & $2.753(8)$ & $\mathrm{O}(40)-\mathrm{O}(56) \# 11$ & $2.746(9)$ \\
$\mathrm{O}(45)-\mathrm{O}(34) \# 1$ & $3.061(10)$ & $\mathrm{O}(50)-\mathrm{O}(54) \# 3$ & $2.793(10)$ \\
$\mathrm{O}(53)-\mathrm{O}(34) \# 12$ & $2.560(18)$ & $\mathrm{O}(54)-\mathrm{O}(22) \# 9$ & $3.079(11)$ \\
$\mathrm{O}(55)-\mathrm{O}(2) \# 13$ & $3.098(13)$ & $\mathrm{O}(5)-\mathrm{O}(56) \# 13$ & $2.702(15)$ \\
$\mathrm{O}(56)-\mathrm{O}(55) \# 5$ & $2.702(15)$ & & \\
\end{tabular}

Symmetry codes are already specified in the footnote Table 5.

at $450 \mathrm{~nm}$ and at around $600 \mathrm{~nm}$, found in the absorption spectrum (Figure 1, right) of compound 2, can be attributed to possible weak spin-forbidden $\mathrm{d}$-d transition in iron ${ }^{32}$ and to the $\mathrm{d}-\mathrm{d}$ transition for the central $\mathrm{Cr}^{3+}$ ion, respectively. The corresponding UV-visible absorption spectra are presented in Figure 1.

\subsection{Powder X-ray diffraction (PXRD)}

The powder X-ray diffraction (PXRD) patterns (Figure 2) of parent compound $\mathbf{1}, \mathbf{1}^{\prime}$ and regenerated $1 \mathrm{did}$ not show any notable change. This may be because of the following reasons: the heated crystals, $\mathbf{1}^{\prime}$ and $\mathbf{1}^{\prime \prime}$ did 
(a)

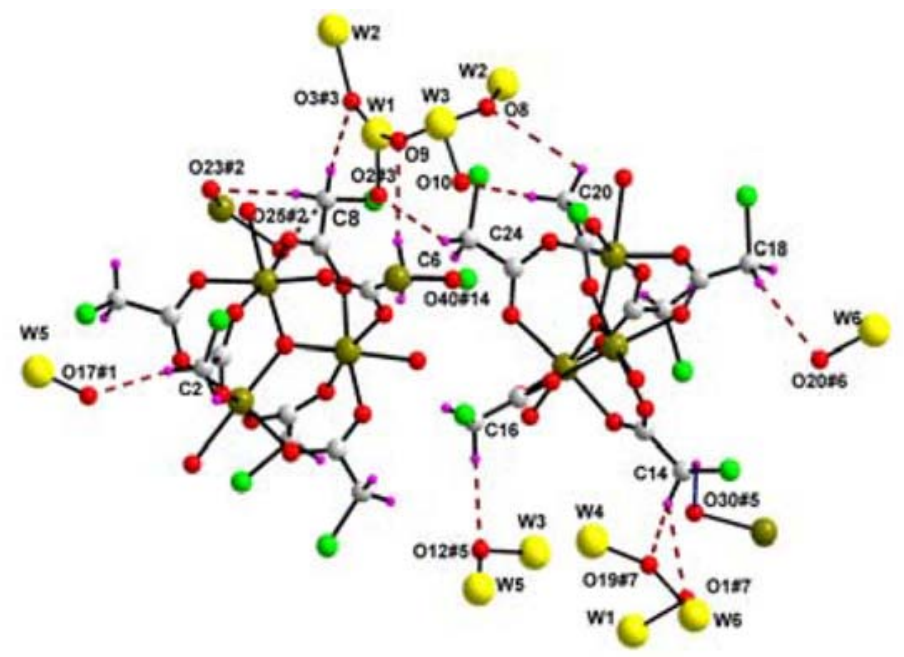

(b)

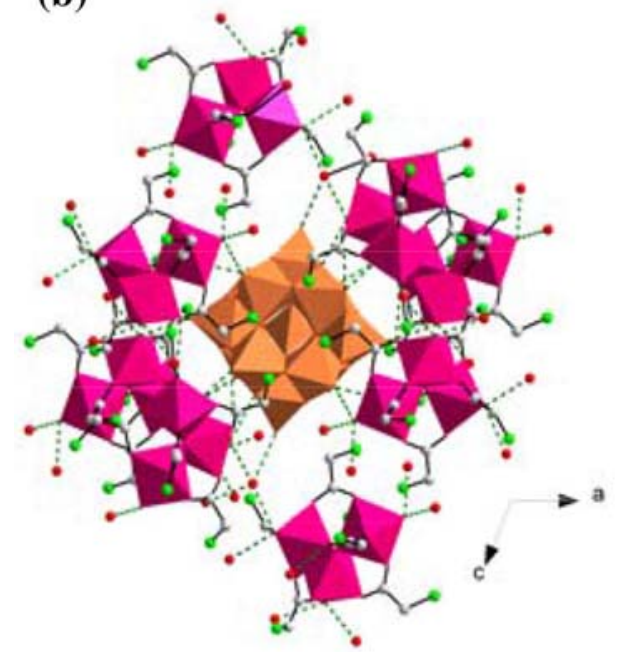

(c)

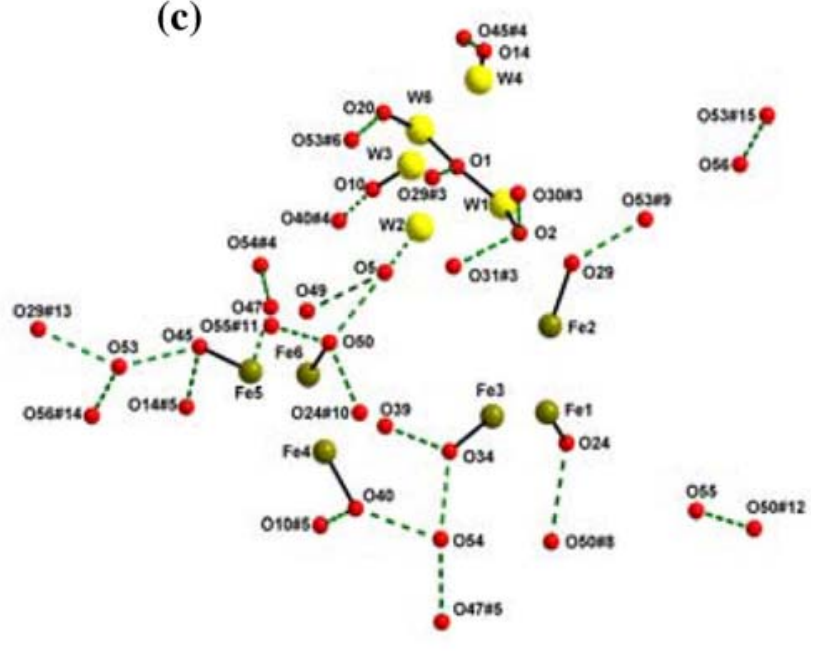

Figure 12. (a) The $\mathrm{C}-\mathrm{H} \cdots \mathrm{O}$ hydrogen bonding interactions in $\left[\mathrm{Fe}_{3}\left(\mu_{3}-\mathrm{O}\right)\left(\mathrm{ClCH}_{2} \mathrm{COO}\right)_{6}\left(\mathrm{H}_{2} \mathrm{O}\right)_{3}\right]_{4}$ $\left[\mathrm{SiW}_{12} \mathrm{O}_{40}\right] \cdot 8 \mathrm{H}_{2} \mathrm{O} \cdot 2 \mathrm{ClCH}_{2} \mathrm{COOH}\left(\mathbf{1}^{\prime \prime}\right)$ with relevant symmetry code. (b) The $\mathrm{O}-\mathrm{H} \cdots \mathrm{O}$ hydrogen bonding interactions in compound $\mathbf{1}^{\prime \prime}$. (c) The $\mathrm{O}-\mathrm{H} \cdots \mathrm{O}$ hydrogen bonding interactions with relevant symmetry codes in compound $\mathbf{1}^{\prime \prime}$. Color code: same as described in caption of Figure 9.

not lose all the lattice water molecules. Indeed, all three systems (parent $\mathbf{1}, \mathbf{1}^{\prime}$ and $\mathbf{1}^{\prime \prime}$ ) have identical unit cell parameters (except the volumes of the unit cells), crystal system and space group (vide infra).

Intentionally, we have avoided higher temperature (for dehydration), because iron-coordinated water molecules are known to leave the crystal lattice resulting in the loss of crystalline state. ${ }^{33,34}$ Similarly, PXRD patterns of compound $\mathbf{2}, \mathbf{2}^{\prime}$ and regenerated $\mathbf{2}$ are also recorded, and as expected, they have identical powder patterns as shown in Figure 2 (right).

\subsection{Thermogravimetric/mass analysis (TGA/mass)}

The thermogravimetric analyses of all the compounds were performed in flowing $\mathrm{N}_{2}$ with a heating rate of $5^{\circ} \mathrm{C}$ $\mathrm{min}^{-1}$ in the temperature range of $30-1100^{\circ} \mathrm{C}$. 3.3a $\left[\mathrm{Fe}_{3}\left(\mu_{3}-\mathrm{O}\right)\left(\mathrm{ClC} \mathrm{H}_{2} \mathrm{COO}\right)_{6}\left(\mathrm{H}_{2} \mathrm{O}\right)_{3}\right]_{4}$ [ $\left.\mathrm{Si} \mathrm{W}_{12} \mathrm{O}_{40}\right] \cdot 18 \mathrm{H}_{2} \mathrm{O} \cdot 2 \mathrm{ClC} \mathrm{H}_{2} \mathrm{COOH}(1)$ : TGA curve of compound 1 is divided into four sets. The first weight loss is $4.18 \%$ that corresponds to the loss of fifteen lattice water molecules per formula unit in the temperature range of $50-120^{\circ} \mathrm{C}$ (calculated mass loss for fifteen water molecules is $4.18 \%$ ). The second weight loss is $3.80 \%$, which corresponds to the loss of fourteen water molecules in the temperature range of $120-170^{\circ} \mathrm{C}$ (calculated mass loss for fourteen water molecules is $3.83 \%$ ). The major mass loss of $37.04 \%$ was observed in the temperature range of $170-585^{\circ} \mathrm{C}$ due to the decomposition of the cluster. The evolution of water molecules (from the lattice and iron coordination sites) and carbon dioxide gas molecules are evidenced by the TGA / Mass curves, which are shown in blue and purple color respectively in Figure 3 (left). 
Table 7. Geometrical parameters of the $\mathrm{C}-\mathrm{H} \cdots \mathrm{O}$ hydrogen bonds $\left(\AA,{ }^{\circ}\right)$ involved in supramolecular network of compound $\left[\mathrm{Fe}_{3}\left(\mu_{3}-\mathrm{O}\right)\left(\mathrm{ClCH}_{2} \mathrm{COO}\right)_{6}\left(\mathrm{H}_{2} \mathrm{O}\right)_{3}\right]_{4}\left[\mathrm{SiW}_{12} \mathrm{O}_{40}\right] \cdot 8 \mathrm{H}_{2} \mathrm{O}$. $2 \mathrm{ClCH}_{2} \mathrm{COOH}\left(\mathbf{1}^{\prime \prime}\right)$. $\mathrm{D}=$ donor; $\mathrm{A}=$ acceptor.

\begin{tabular}{|c|c|c|c|c|}
\hline D-H $\cdots$ A & $\mathrm{d}(\mathrm{D} \cdots \mathrm{H})$ & $\mathrm{d}(\mathrm{H} \cdots \mathrm{A})$ & $\mathrm{d}(\mathrm{D} \cdots \mathrm{A})$ & $\angle$ (DHA \\
\hline C2-H2B....O17\#1 & 0.97 & 2.52 & $3.400(2)$ & 151.5 \\
\hline C6-H6B....O9 & 0.97 & 2.64 & $3.545(16)$ & 156.3 \\
\hline С6-Н6А...О40\#4 & 0.97 & 2.79 & $3.358(18)$ & 118.0 \\
\hline C8-H8A....О3\#3 & 0.97 & 2.41 & $3.375(16)$ & 173.2 \\
\hline $\mathrm{C} 8-\mathrm{H} 8 \mathrm{~B} \ldots . . \mathrm{O} 23 \# 2$ & 0.97 & 2.54 & $3.498(19)$ & 170.5 \\
\hline $\mathrm{C} 8-\mathrm{H} 8 \mathrm{~B} \ldots . . \mathrm{O} 25 \# 2$ & 0.97 & 2.84 & $3.410(19)$ & 118.1 \\
\hline C14-H14B...O19\#7 & 0.97 & 2.82 & $3.480(17)$ & 125.6 \\
\hline C14-H14B....O1\#7 & 0.97 & 2.56 & $3.385(17)$ & 143.5 \\
\hline C14-H14A....О30\#5 & 0.97 & 2.57 & $3.328(16)$ & 134.7 \\
\hline С16-H16А....O12\#5 & 0.97 & 2.47 & $3.438(17)$ & 177.8 \\
\hline C18-H18B....O20\#6 & 0.97 & 2.61 & $3.221(18)$ & 120.7 \\
\hline C20-H20A....O10 & 0.97 & 2.29 & $3.116(17)$ & 142.1 \\
\hline C20-H20A....O & 0.97 & 2.73 & $3.387(16)$ & 125.5 \\
\hline C24-H24A....O2\#3 & 0.97 & 2.63 & $3.490(2)$ & 147.8 \\
\hline
\end{tabular}

Symmetry transformations used to generate equivalent atoms: \#1 x, -y, 0.5+z; \#2 1-x, 1-y, 1-z; \#3 1-x, y, 0.5-z; \#4 0.5-x, -0.5+y, 0.5-z; \#5 0.5-x, 0.5+y, 0.5-z; \#6 0.5-x, 0.5-y, -z; \#7 -0.5+x, 0.5+y, z; \#8 x, 1-y, 0.5+z; \#9 $0.5+x, 0.5-y, 0.5+z ; \# 10 \mathrm{x}, 1-\mathrm{y},-0.5+\mathrm{z}$; \#11 x, y, -1+z; \#12 x, y, 1+z; \#13 -0.5+x, 0.5-y, -0.5+z; \#14 -1+x, y, z; \#15 1+x, y, z.

Table 8. Geometrical parameters of the $\mathrm{O}-\mathrm{H} \cdots \mathrm{O}$ hydrogen bonds $(\AA)$ involved in supramolecular network of compound $\left[\mathrm{Fe}_{3}\left(\mu_{3}-\mathrm{O}\right)\left(\mathrm{ClCH}_{2} \mathrm{COO}\right)_{6}\left(\mathrm{H}_{2} \mathrm{O}\right)_{3}\right]_{4}\left[\mathrm{SiW}_{12} \mathrm{O}_{40}\right] \cdot 8 \mathrm{H}_{2} \mathrm{O}$. $2 \mathrm{ClCH}_{2} \mathrm{COOH}\left(\mathbf{1}^{\prime \prime}\right)$.

\begin{tabular}{lccc}
\hline $\mathrm{O}(2)-\mathrm{O}(30) \# 3$ & $2.900(12)$ & $\mathrm{O}(2)-\mathrm{O}(31) \# 3$ & $3.035(14)$ \\
$\mathrm{O}(1)-\mathrm{O}(29) \# 3$ & $2.727(12)$ & $\mathrm{O}(5)-\mathrm{O}(49)$ & $3.087(13)$ \\
$\mathrm{O}(5)-\mathrm{O}(50)$ & $2.849(13)$ & $\mathrm{O}(10)-\mathrm{O}(40) \# 4$ & $2.734(12)$ \\
$\mathrm{O}(14)-\mathrm{O}(45) \# 4$ & $2.898(12)$ & $\mathrm{O}(20)-\mathrm{O}(53) \# 6$ & $3.069(14)$ \\
$\mathrm{O}(24)-\mathrm{O}(50) \# 8$ & $3.038(14)$ & $\mathrm{O}(29)-\mathrm{O}(53) \# 9$ & $2.733(13)$ \\
$\mathrm{O}(34)-\mathrm{O}(39)$ & $2.866(14)$ & $\mathrm{O}(34)-\mathrm{O}(54)$ & $2.773(14)$ \\
$\mathrm{O}(40)-\mathrm{O}(10) \# 5$ & $2.734(12)$ & $\mathrm{O}(40)-\mathrm{O}(54)$ & $2.768(14)$ \\
$\mathrm{O}(45)-\mathrm{O}(14) \# 5$ & $2.898(12)$ & $\mathrm{O}(45)-\mathrm{O}(53)$ & $2.717(14)$ \\
$\mathrm{O}(47)-\mathrm{O}(54) \# 4$ & $3.048(14)$ & $\mathrm{O}(50)-\mathrm{O}(24) \# 10$ & $3.038(14)$ \\
$\mathrm{O}(50)-\mathrm{O}(55) \# 11$ & $2.500(3)$ & $\mathrm{O}(55)-\mathrm{O}(50) \# 12$ & $2.500(3)$ \\
$\mathrm{O}(53)-\mathrm{O}(29) \# 13$ & $2.733(13)$ & $\mathrm{O}(53)-\mathrm{O}(56) \# 14$ & $2.710(2)$ \\
$\mathrm{O}(54)-\mathrm{O}(47) \# 5$ & $3.048(14)$ & $\mathrm{O}(56)-\mathrm{O}(53) \# 15$ & $2.710(2)$ \\
\hline
\end{tabular}

Symmetric codes are already specified in the footnote of Table 7.

3.3b $\left[\mathrm{Fe}_{3}\left(\mu_{3}-\mathrm{O}\right)\left(\mathrm{ClC} \mathrm{H}_{2} \mathrm{COO}\right)_{6}\left(\mathrm{H}_{2} \mathrm{O}\right)_{3}\right]_{4}$ $\left[\mathrm{SiW}_{12} \mathrm{O}_{40}\right] \cdot 10 \mathrm{H}_{2} \mathrm{O} \cdot 2 \mathrm{ClCH}_{2} \mathrm{COOH}\left(\mathbf{1}^{\prime}\right):$ TGA curve (Figure 3, right) of compound $\mathbf{1}^{\prime}$ is divided into three sets. The first weight loss $1.90 \%$, which corresponds to the loss of seven lattice water molecules per formula unit in the temperature range of $50-140^{\circ} \mathrm{C}$ (calculated mass loss for seven water molecules is $1.96 \%$ ). The second weight loss is $3.96 \%$ corresponding to the loss of fourteen water molecules in the temperature range of $140-201^{\circ} \mathrm{C}$ (calculated mass loss for fourteen water molecules is $3.91 \%$ ). The major mass loss of $38.67 \%$ was observed in the temperature range of 201$748^{\circ} \mathrm{C}$ due to the collapse of the structure of the cluster. The evolution of water molecules and carbon dioxide gas molecules are evidenced by the TGA / Mass curves, which are shown in blue and purple color respectively in the Figure 3, right $\left(\mathbf{1}^{\prime}\right)$.

3.3c $\left[\mathrm{Fe}_{3}\left(\mu_{3} \mathrm{O}\right)\left(\mathrm{ClC} \mathrm{H}_{2} \mathrm{COO}\right)_{6}\left(\mathrm{H}_{2} \mathrm{O}\right)_{3}\right]_{4}$ [ $\left.\mathrm{Si} \mathrm{W}_{12} \mathrm{O}_{40}\right] \cdot 8 \mathrm{H}_{2} \mathrm{O} \cdot 2 \mathrm{ClC} \mathrm{H}_{2} \mathrm{COOH}\left(\mathbf{1}^{\prime \prime}\right)$ : TGA curve 
Table 9. Crystal Data and Structural Refinement for Compounds 2, dehydrated 2-85 ${ }^{\circ}\left(2^{\prime}\right)$ and dehydrated $2-\mathbf{1 3 5}^{\circ}\left(\mathbf{2}^{\prime}\right)$.

\begin{tabular}{|c|c|c|c|}
\hline & 2 & 2 dehydrated $2-85^{\circ} 2^{\prime}$ & dehydrated $2-135^{\circ} 2^{\prime}$ \\
\hline \multirow[t]{2}{*}{ Empirical formula } & $\mathrm{C}_{52} \mathrm{H}_{106} \mathrm{Cl}_{26} \mathrm{Cr}_{12}$ & $\mathrm{C}_{52} \mathrm{H}_{94} \mathrm{Cl}_{26} \mathrm{Cr}_{12}$ & $\mathrm{C}_{52} \mathrm{H}_{94} \mathrm{Cl}_{26} \mathrm{Cr}_{12}$ \\
\hline & $\mathrm{O}_{122} \mathrm{SiW}_{12}$ & $\mathrm{O}_{116} \mathrm{SiW}_{12}$ & $\mathrm{O}_{116} \mathrm{SiW}_{12}$ \\
\hline Formula weight & 6463.36 & 6355.26 & 6355.26 \\
\hline $\mathrm{T}[\mathrm{K}]$ & 293(2) & 293(2) & $293(2)$ \\
\hline$\lambda[\AA]$ & 0.71073 & 0.71073 & 0.71073 \\
\hline Crystal system & Monoclinic & Monoclinic & Monoclinic \\
\hline Space group & $C 2 / c$ & $C 2 / c$ & $C 2 / c$ \\
\hline$a[\AA]$ & $30.1958(17)$ & $30.539(6)$ & $30.569(8)$ \\
\hline$b[\AA]$ & $17.7025(10)$ & $17.777(3)$ & $17.778(4)$ \\
\hline$c[\AA]$ & $32.2944(18)$ & $29.880(6)$ & $29.823(7)$ \\
\hline$\alpha[\mathrm{deg}]$ & 90.0000 & 90.000 & 90.000 \\
\hline$\beta$ [deg] & $101.6470(10)$ & $112.962(3)$ & $112.809(4)$ \\
\hline$\gamma[\mathrm{deg}]$ & 90.0000 & 90.000 & 90.000 \\
\hline$V\left[\AA^{3}\right]$ & $16907.2(16)$ & $14936(5)$ & $14940(6)$ \\
\hline$Z$ & 4 & 4 & 4 \\
\hline $\mathrm{D}_{\text {calc }}\left[\mathrm{Mg} \mathrm{m}^{-3}\right]$ & 2.539 & 2.826 & 2.825 \\
\hline$\mu\left[\mathrm{mm}^{-1}\right]$ & 9.386 & 10.619 & 10.616 \\
\hline $\mathrm{F}[000]$ & 12104 & 11864 & 11864 \\
\hline Crystal size $\left[\mathrm{mm}^{3}\right]$ & $0.26 \times 0.10 \times 0.06$ & $0.22 \times 0.10 \times 0.06$ & $0.30 \times 0.10 \times 0.02$ \\
\hline $\begin{array}{l}\theta \text { range for data } \\
\text { collection [deg] }\end{array}$ & 1.34 to 26.05 & 1.48 to 28.36 & 1.45 to 26.54 \\
\hline Reflections & $1.04+1020.03$ & 1.40620 .50 & 1.451020 .04 \\
\hline Collected/unique & $87235 / 16671$ & $85248 / 17891$ & $69592 / 14651$ \\
\hline $\mathrm{R}$ [int] & 0.0473 & 0.0674 & 0.1329 \\
\hline Refinement method & Full-matrix least-squares on $\mathrm{F}^{2}$ & & \\
\hline Data/restraints/parameters & $16671 / 0 / 1029$ & $17891 / 0 / 964$ & $14651 / 0 / 953$ \\
\hline Goodness-of-fit on $\mathrm{F}^{2}$ & 1.048 & 1.182 & 0.962 \\
\hline $\mathrm{R}_{1} / \mathrm{wR}_{2}[\mathrm{I}>2 \sigma(\mathrm{I})]$ & $0.0354 / 0.0911$ & $0.0619 / 0.1226$ & $0.0470 / 0.0946$ \\
\hline $\mathrm{R}_{1} / \mathrm{wR}_{2}$ (all data) & $0.0452 / 0.0959$ & $0.0779 / 0.1286$ & $0.0809 / 0.1048$ \\
\hline Largest diff. Peak/hole $\left[\mathrm{e} \AA^{-3}\right.$ ] & $2.125 /-1.308$ & $2.711 /-2.473$ & $2.038 /-1.843$ \\
\hline
\end{tabular}

of dehydrated $\mathbf{1}^{\prime \prime}$ can be divided into four sets. The first weight loss of $2.08 \%$ corresponds to the loss of seven lattice water molecules per formula unit in the temperature range of $50-140^{\circ} \mathrm{C}$ (calculated mass loss for seven water molecules is $1.97 \%$ ). The second weight loss of $3.62 \%$ corresponds to the loss of thirteen water molecules in the temperature range of $140-201^{\circ} \mathrm{C}$ (calculated mass loss for thirteen water molecules is $3.66 \%$ ). Two major mass losses $(27.22 \%$ and $7.77 \%)$ are observed in the temperature range of $207-598^{\circ} \mathrm{C}$, due to the decomposition of the structure of the trinuclear iron cluster. The evolution of water molecules and carbon dioxide gas molecules are shown by the TGA / Mass curves, which are shown in blue and purple color, respectively in the Figure 4, left.

\section{3d $\left[\mathrm{Fe}_{3}\left(\mu_{3}-\mathrm{O}\right)\left(\mathrm{ClC} \mathrm{H}_{2} \mathrm{COO}\right)_{6}\left(\mathrm{H}_{2} \mathrm{O}\right)_{3}\right]_{4}$} [ $\left.\mathrm{Si}_{12} \mathrm{O}_{40}\right] \cdot 18 \mathrm{H}_{2} \mathrm{O} \cdot 2 \mathrm{ClC}_{2} \mathrm{COOH}($ regenerated 1): The bunch of crystals of regenerated 1 was dried in room temperature and then loaded in to thermogravimetric crucible for TGA/Mass analysis. The typical TGA curve has been divided into three groups. The first weight loss of $2.89 \%$ corresponds to the loss of eleven lattice water molecules per formula unit in the temperature range of $50-120^{\circ} \mathrm{C}$ (calculated mass loss for eleven water molecules is $3.01 \%$ ). The water mass loss in this stage is significantly less than the expected $4.18 \%$ (from parent compound 1, Figure 3(1)). This might be due to the removal of some water molecules (approximately four water molecules) during the drying process of the relevant crystals at room temperature. The second weight loss of $3.78 \%$ corresponds to the loss of fourteen water molecules in the temperature range of $120-181^{\circ} \mathrm{C}$ (calculated mass loss for fourteen water molecules is $3.83 \%)$. The major mass loss $(35.02 \%)$ is observed in the temperature range of $181-506^{\circ} \mathrm{C}$ due to the decomposition of the pertinent cluster. The evolution of water molecules and carbon dioxide gas molecules 


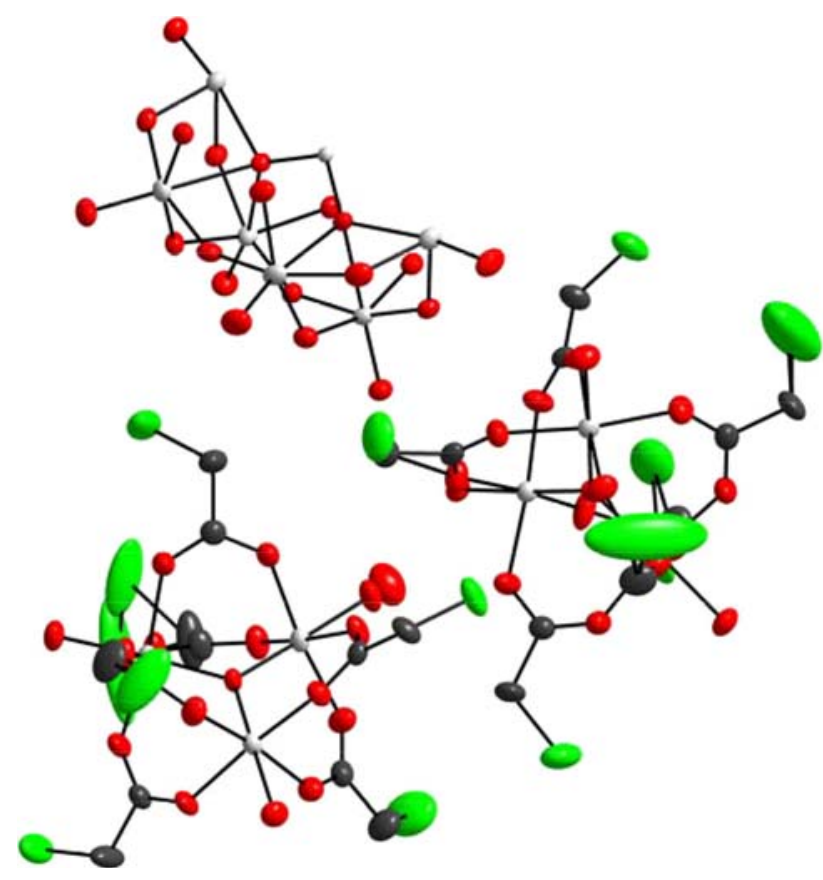

Figure 13. Thermal ellipsoidal plot of the asymmetric unit of compound 2 (50\% probability). Hydrogen atoms, lattice water molecules and lattice chloroacetic acid were omitted for clarity.

are observed by the TGA/Mass curves which are shown in blue and purple color respectively in the Figure 4, right.

\section{3e $\left[\mathrm{Cr}_{3}\left(\mu_{3}-\mathrm{O}\right)\left(\mathrm{ClCH}_{2} \mathrm{COO}\right)_{6}\left(\mathrm{H}_{2} \mathrm{O}\right)_{3}\right]_{4}$}

[ $\left.\mathrm{Si} \mathrm{W}_{12} \mathrm{O}_{40}\right] \cdot 14 \mathrm{H}_{2} \mathrm{O} \cdot 2 \mathrm{ClCH}_{2} \mathrm{COOH}(2)$ : TGA curve (Figure 5, left) of compound $\mathbf{2}$ is divided into three sets. The first weight loss of $2.63 \%$ corresponds to the loss of nine lattice water molecules per formula unit in the temperature range of $50-130^{\circ} \mathrm{C}$ (calculated mass loss for nine water molecules is $2.51 \%$ ). The second weight loss of $3.51 \%$, which corresponds to the loss of thirteen water molecules (five water molecules from lattice and eight water molecules from iron coordination sites), occurred in the temperature range of $130-220^{\circ} \mathrm{C}$ (calculated mass loss for fifteen water molecules is $3.62 \%$ ). The major weight loss of $37.60 \%$ was observed in the temperature range of $220-670^{\circ} \mathrm{C}$ showing the decomposition of the concerned cluster. The evolution of water molecules and carbon dioxide gas molecules are evidenced by the TGA/Mass curves, which are shown in blue and purple color respectively in the Figure 5, left.

\section{3f $\left[\mathrm{Cr}_{3}\left(\mu_{3}-\mathrm{O}\right)\left(\mathrm{ClC} \mathrm{H}_{2} \mathrm{COO}\right)_{6}\left(\mathrm{H}_{2} \mathrm{O}\right)_{3}\right]_{4}$} $\left[\mathrm{Si} \mathrm{W}_{12} \mathrm{O}_{40}\right] \cdot 8 \mathrm{H}_{2} \mathrm{O} \cdot 2 \mathrm{ClC} \mathrm{H}_{2} \mathrm{COOH}$ (dehydrated 2$85^{\circ} \mathrm{C} / 135^{\circ} \mathrm{C} \equiv \mathbf{2}^{\prime}$ ): TGA curve (Figure 5, right) of $\mathbf{2}^{\prime}$ is divided into four portions. The first weight loss of $1.83 \%$, which corresponds to the loss of six lattice water molecules per formula unit, occurred in the temperature range of $50-130^{\circ} \mathrm{C}$ (calculated mass loss for six water molecules is $1.70 \%$ ). The second weight loss of $3.87 \%$ corresponds to the loss of fourteen water molecules (eight water molecules from lattice and six water molecules from iron coordination sites) in the temperature range of $142-191{ }^{\circ} \mathrm{C}$ (calculated mass loss for fourteen water molecules is $3.97 \%$ ). Two major weight losses $(28.71 \%$ and $11.54 \%)$ were observed in the temperature range of $240-1017^{\circ} \mathrm{C}$ due to decomposition of the cluster. The evolution of water molecules and carbon dioxide gas molecules are evidenced in the TGA/Mass curves that are shown in blue and purple colors respectively as shown in the Figure 5, right.

\section{$3.3 \mathrm{~g}\left[\mathrm{Cr}_{3}\left(\mu_{3}-\mathrm{O}\right)\left(\mathrm{ClC} \mathrm{H}_{2} \mathrm{COO}\right)_{6}\left(\mathrm{H}_{2} \mathrm{O}\right)_{3}\right]_{4}$} [ $\left.\mathrm{Si} \mathrm{W}_{12} \mathrm{O}_{40}\right] \cdot 14 \mathrm{H}_{2} \mathrm{O} \cdot 2 \mathrm{ClC}_{2} \mathrm{COOH}($ regenerated 2): The bunch of crystals of regenerated 2 was dried at room temperature and then the powdered sample was loaded in thermogravimetric crucible for TGA/Mass analysis.

The typical TGA curve has been divided into four groups (Figure 6). The first weight loss of $3.02 \%$ corresponds to the loss of eleven lattice water molecules per formula unit in the temperature range of $50-100^{\circ} \mathrm{C}$ (calculated mass loss for eleven water molecules is $3.06 \%$ ). The mass loss in regenerated 2 (3.02\% for eleven lattice water molecules) in this stage is approximately comparable to the mass loss obtained in parent compound 2 where the same was $2.63 \%$ for nine lattice water molecules). The second weight loss of $3.90 \%$ corresponds to the loss of fourteen water molecules in the temperature range of $100-190^{\circ} \mathrm{C}$ (calculated mass loss for fourteen water molecules is $3.90 \%$ ). The major mass losses of $32.90 \%$ and $7.52 \%$, observed in the temperature range of $190-1100^{\circ} \mathrm{C}$, are due to the decomposition of the cluster structure. The evolution of water molecules and carbon dioxide gas molecules are confirmed by the TGA/Mass curves that are shown in blue and purple color respectively as shown in Figure 6.

\section{$3.4 X$-ray crystallographic studies}

\section{4a $\left[\mathrm{Fe}_{3}\left(\mu_{3}-\mathrm{O}\right)\left(\mathrm{ClCH}_{2} \mathrm{COO}\right)_{6}\left(\mathrm{H}_{2} \mathrm{O}\right)_{3}\right]_{4}$}

[ $\left.\mathrm{Si} \mathrm{W}_{12} \mathrm{O}_{40}\right] \cdot 18 \mathrm{H}_{2} \mathrm{O} \cdot 2 \mathrm{ClC} \mathrm{H}_{2} \mathrm{COOH}(\mathbf{1})$ : Compound 1 crystallizes in monoclinic system with space group $C 2 / c$ and single crystal X-ray data, obtained at 298 $\mathrm{K}$, are summarized in Table 1 . In compound $\mathbf{1}$, the asymmetric unit contains half part of Keggin anion (in which $\mathrm{Si}$ atom has half occupancy), two basic trinuclear iron-oxo macrocations, one lattice $\mathrm{ClCH}_{2} \mathrm{COOH}$ and nine non-coordinated (lattice) water molecules. 
(a)

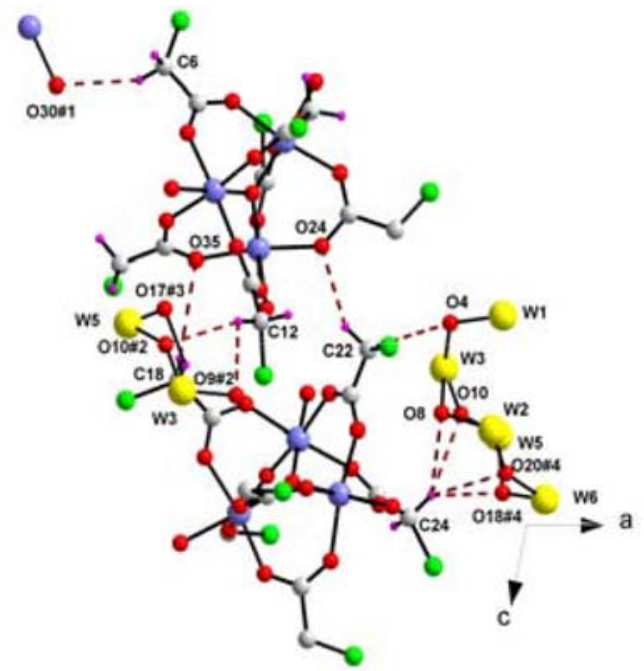

(b)

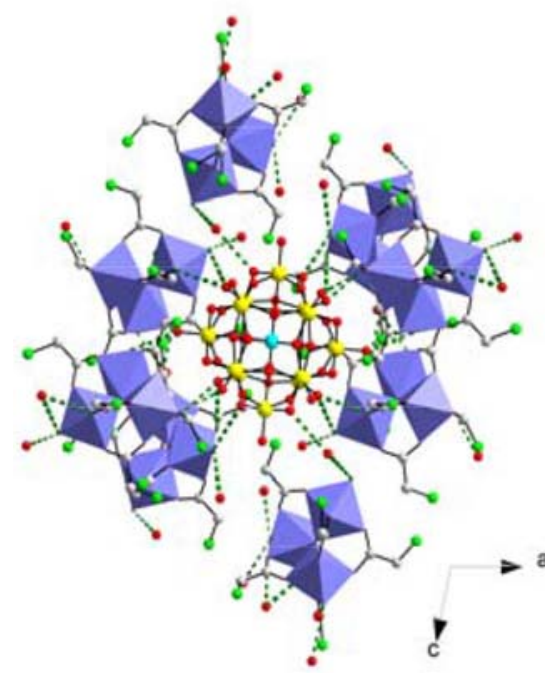

(c)
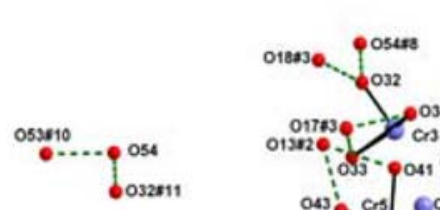

Figure 14. (a) $\mathrm{C}-\mathrm{H} \cdots \mathrm{O}$ hydrogen bonding interactions in $\left[\mathrm{Cr}_{3}\left(\mu_{3}-\mathrm{O}\right)\left(\mathrm{ClCH}_{2} \mathrm{COO}\right)_{6}\left(\mathrm{H}_{2} \mathrm{O}\right)_{3}\right]_{4}\left[\mathrm{SiW}_{12}\right.$ $\left.\mathrm{O}_{40}\right] \cdot 14 \mathrm{H}_{2} \mathrm{O} \cdot 2 \mathrm{ClCH}_{2} \mathrm{COOH}(2)$ along with relevant symmetry code. (b) $\mathrm{O}-\mathrm{H} \cdots \mathrm{O}$ Hydrogen bonding interactions in compound 2; (c) $\mathrm{O}-\mathrm{H} \cdots \mathrm{O}$ hydrogen bonding interactions with relevant symmetry codes in compound 2. Color code: W, yellow; $\mathrm{Si}$, cyan; $\mathrm{O}$, red; $\mathrm{Cr}$, violet; $\mathrm{Cl}$, light green; $\mathrm{C}$, gray; $\mathrm{H}$, purple; $\left[\mathrm{Cr}_{3}\left(\mu_{3}-\mathrm{O}\right)\left(\mathrm{ClCH}_{2} \mathrm{COO}\right)_{6}\left(\mathrm{H}_{2} \mathrm{O}\right)_{3}\right]^{1+}$ cluster, violet polyhedra; $\mathrm{C}-\mathrm{H} \cdots \mathrm{O}$ hydrogen bonding interactions, brown dotted lines; $\mathrm{O}-\mathrm{H} \cdots \mathrm{O}$ hydrogen bonding interactions green dotted lines.

Accordingly, compound 1 can be formulated as $\left[\mathrm{Fe}_{3}\left(\mu_{3}\right.\right.$ $\left.\mathrm{O})\left(\mathrm{ClCH}_{2} \mathrm{COO}\right)_{6}\left(\mathrm{H}_{2} \mathrm{O}\right)_{3}\right]_{4}\left[\mathrm{SiW}_{12} \mathrm{O}_{40}\right] \cdot 18 \mathrm{H}_{2} \mathrm{O} \cdot 2 \mathrm{ClCH}_{2}$ $\mathrm{COOH}(\mathbf{1})$. The thermal ellipsoidal plot of the asymmetric unit of compound $\mathbf{1}$ is presented in Figure 7(a) (the solvent water molecules and lattice chloroacetic acid are not shown). The molecular structure of compound $\mathbf{1}$ is shown in Figure 7(b). The structure of Keggin anion can be described as four $\mathrm{W}_{3} \mathrm{O}_{13}$ groups surrounding the central hetero atom $\mathrm{Si}$. Each $\mathrm{W}_{3} \mathrm{O}_{13}$ group is formed by three $\mathrm{WO}_{6}$ octahedra sharing edges and having a common oxygen atom which is also shared by the central hetero atom. On the whole, the oxygen bonding modes in Keggin (POM) anion can be grouped into four different categories: (i) $\mathrm{W}-\mathrm{O}_{\mathrm{t}}\left(\mathrm{O}_{\mathrm{t}}\right.$ is terminal oxygen) $1.694(7)$
$-1.716(7) \AA$; (ii) $\mathrm{W}-\mathrm{O}\left(\mu_{2}\right), 1.894(7)-1.950(7) \AA$; (iii) $\mathrm{W}-\mathrm{O}\left(\mu_{4}\right), 2.333(6)-2.360(6) \AA$; (iv) $\mathrm{Si}-\mathrm{O}\left(\mu_{4}\right)$, $1.625(6)-1.627(6) \AA$.

The structure of $\left[\mathrm{Fe}_{3}\left(\mu_{3}-\mathrm{O}\right)\left(\mathrm{ClCH}_{2} \mathrm{COO}\right)_{6}\left(\mathrm{H}_{2} \mathrm{O}\right)_{3}\right]^{1+}$ macrocation can be described as follows: Each $\mathrm{ClCH}_{2}$ $\mathrm{COOH}$ bridges two adjacent iron metal centers in bidentate fashion through carboxylate functionality and every two adjacent iron metal centers are bridged by two such bidentate ligands. Further, the terminal position of each metal center is occupied by a monodentate water molecule. All three iron metal ions $\left(\mathrm{Fe}^{3+}\right)$ are bridged by a triply bridging oxygen atom. The geometry around each iron metal center is octahedral and the overall charge of each macrocation is +1 . In the molecular struc- 
Table 10. Geometrical parameters of the $\mathrm{C}-\mathrm{H} \cdots \mathrm{O}$ hydrogen bonds $\left(\AA,{ }^{\circ}\right)$ involved in supramolecular network of compound $\left[\mathrm{Cr}_{3}\left(\mu_{3}-\mathrm{O}\right)\left(\mathrm{ClCH}_{2} \mathrm{COO}\right)_{6}\left(\mathrm{H}_{2} \mathrm{O}\right)_{3}\right]_{4}\left[\mathrm{SiW}_{12} \mathrm{O}_{40}\right] \cdot 14 \mathrm{H}_{2} \mathrm{O}$. $2 \mathrm{ClCH}_{2} \mathrm{COOH}$ (2). $\mathrm{D}=$ donor; $\mathrm{A}=$ acceptor.

\begin{tabular}{|c|c|c|c|c|}
\hline $\mathrm{D}-\mathrm{H} \cdot \mathrm{A}$ & $\mathrm{d}(\mathrm{D} \cdots \mathrm{H})$ & $\mathrm{d}(\mathrm{H} \cdots \mathrm{A})$ & $\mathrm{d}(\mathrm{D} \cdots \mathrm{A})$ & $\angle$ (DHA \\
\hline С6-H6В....О30\#1 & 0.97 & 2.60 & $3.383(11)$ & 137.9 \\
\hline C12-H12A...O9\#2 & 0.97 & 2.90 & $3.472(9)$ & 118.8 \\
\hline C12-H12A....O10\#2 & 0.97 & 2.57 & $3.475(10)$ & 155.9 \\
\hline C18-H18B....O17\#3 & 0.97 & 2.62 & $3.494(12)$ & 150.7 \\
\hline C18-H18A....O35 & 0.97 & 2.96 & $3.587(11)$ & 123.3 \\
\hline C22-H22B...O4 & 0.97 & 2.41 & $3.382(9)$ & 177.2 \\
\hline C22-H22A....O24 & 0.97 & 2.70 & $3.547(10)$ & 145.7 \\
\hline C24-H24A....O8 & 0.97 & 2.95 & $3.527(10)$ & 118.9 \\
\hline C24-H24A...O 10 & 0.97 & 2.69 & $3.594(10)$ & 154.4 \\
\hline C24-H24A....O18\#4 & 0.97 & 2.59 & $3.374(10)$ & 138.6 \\
\hline C24-H24A....O20\#4 & 0.97 & 2.74 & $3.444(10)$ & 130.3 \\
\hline
\end{tabular}

Symmetry transformations used to generate equivalent atoms: \#1 -x, 1-y, -z; \#2 0.5-x, 0.5+y, 0.5-z; \#3 -0.5+x, 0.5+y, z; \#4 1-x, y, 0.5-z; \#5 0.5-x, -0.5+y, 0.5-z; \#6 0.5-x, 0.5-y, 1-z; \#7 0.5+x, -0.5+y, z; \#8 x, y, -1+z; \#9 1+x, y, z; \#10 -1+x, y, z; \#11 x, y, 1+z.

Table 11. Geometrical parameters of the $\mathrm{O}-\mathrm{H} \cdots \mathrm{O}$ hydrogen bonds $(\AA)$ involved in supramolecular network of compound $\left[\mathrm{Cr}_{3}\left(\mu_{3}-\mathrm{O}\right)\left(\mathrm{ClCH}_{2} \mathrm{COO}\right)_{6}\left(\mathrm{H}_{2} \mathrm{O}\right)_{3}\right]_{4}\left[\mathrm{SiW}_{12} \mathrm{O}_{40}\right] \cdot 14 \mathrm{H}_{2} \mathrm{O}$. $2 \mathrm{ClCH}_{2} \mathrm{COOH}(2)$.

\begin{tabular}{lccc}
\hline $\mathrm{O}(7)-\mathrm{O}(38)$ & $2.912(7)$ & $\mathrm{O}(9)-\mathrm{O}(48)$ & $2.726(7)$ \\
$\mathrm{O}(13)-\mathrm{O}(41) \# 5$ & $2.992(7)$ & $\mathrm{O}(13)-\mathrm{O}(43) \# 5$ & $3.003(8)$ \\
$\mathrm{O}(15)-\mathrm{O}(55) \# 6$ & $3.069(10)$ & $\mathrm{O}(17)-\mathrm{O}(33) \# 7$ & $2.899(7)$ \\
$\mathrm{O}(17)-\mathrm{O}(34) \# 7$ & $2.890(7)$ & $\mathrm{O}(18)-\mathrm{O}(32) \# 7$ & $2.909(7)$ \\
$\mathrm{O}(22)-\mathrm{O}(46)$ & $2.900(8)$ & $\mathrm{O}(22)-\mathrm{O}(58)$ & $2.712(9)$ \\
$\mathrm{O}(27)-\mathrm{O}(55) \# 8$ & $2.715(11)$ & $\mathrm{O}(32)-\mathrm{O}(54) \# 8$ & $2.740(2)$ \\
$\mathrm{O}(32)-\mathrm{O}(18) \# 3$ & $2.909(7)$ & $\mathrm{O}(33)-\mathrm{O}(17) \# 3$ & $2.899(7)$ \\
$\mathrm{O}(34)-\mathrm{O}(17) \# 3$ & $2.890(7)$ & $\mathrm{O}(38)-\mathrm{O}(56)$ & $2.638(12)$ \\
$\mathrm{O}(41)-\mathrm{O}(13) \# 2$ & $2.992(7)$ & $\mathrm{O}(43)-\mathrm{O}(13) \# 2$ & $3.003(8)$ \\
$\mathrm{O}(43)-\mathrm{O}(57)$ & $2.677(13)$ & $\mathrm{O}(48)-\mathrm{O}(58)$ & $2.749(9)$ \\
$\mathrm{O}(53)-\mathrm{O}(54) \# 9$ & $2.680(5)$ & $\mathrm{O}(54)-\mathrm{O}(53) \# 10$ & $2.680(5)$ \\
$\mathrm{O}(54)-\mathrm{O}(32) \# 11$ & $2.740(2)$ & $\mathrm{O}(55)-\mathrm{O}(27) \# 11$ & $2.715(11)$ \\
\hline
\end{tabular}

Symmetric codes are already specified in the footnote of Table 10.

ture of compound $\mathbf{1}$, four macrocations neutralize the charge of a Keggin anion $\left[\mathrm{SiW}_{12} \mathrm{O}_{40}\right]^{4-}$. Compound $\mathbf{1}$ contains two lattice $\mathrm{ClCH}_{2} \mathrm{COOH}$ and eighteen lattice water molecules per formula unit. Out of these lattice water molecules, two types of water clusters, namely water trimer (water molecules O53, O56 and O58, $\mathrm{O}$. . O separation distance is in the range of 2.820(3) - 2.910(3) ^) and water dimer (water molecules O57 and O60, O . . O separation distance is 3.030(5) $\AA$ ) are observed in the crystal lattice of compound 1 (located in hydrophilic channels) as shown in Figure 8(a). Lattice $\mathrm{ClCH}_{2} \mathrm{COOH}$ is considerably disordered and hence refined isotorpically only. The overall structure is stabilized by the electrostatic interactions between $\left[\mathrm{Fe}_{3}\left(\mu_{3}-\mathrm{O}\right)\left(\mathrm{ClCH}_{2} \mathrm{COO}\right)_{6}\left(\mathrm{H}_{2} \mathrm{O}\right)_{3}\right]^{1+}$ macrocation and
$\left[\mathrm{SiW}_{12} \mathrm{O}_{40}\right]^{4-}$ cluster anion. Further, the supramolecular $\mathrm{C}-\mathrm{H} \cdots \mathrm{O}$ and $\mathrm{O}-\mathrm{H} \cdots \mathrm{O}$ hydrogen bonding interactions offer extra stability in obtaining this ion pair system. The possible $\mathrm{C}-\mathrm{H} \cdots \mathrm{O}$ and $\mathrm{O}-\mathrm{H} \cdots \mathrm{O}$ hydrogen bonding interactions are shown in Figure 9. The relevant hydrogen bonding parameters (for $\mathrm{C}-\mathrm{H} \ldots \mathrm{O}$ interactions, D . . A distance is considered up to $3.6 \AA$, whereas for $\mathrm{O}-\mathrm{H} \cdots \mathrm{O}$ interactions, the same is considered up to $3.1 \AA$ ) are presented in Tables 2 and 3.

\subsection{Water exclusion (dehydration) and inclusion (regeneration) experiments in compound 1}

Compound 1 originally contains eighteen lattice water molecules. When the crystals of compound $\mathbf{1}$ are heated 
(a)

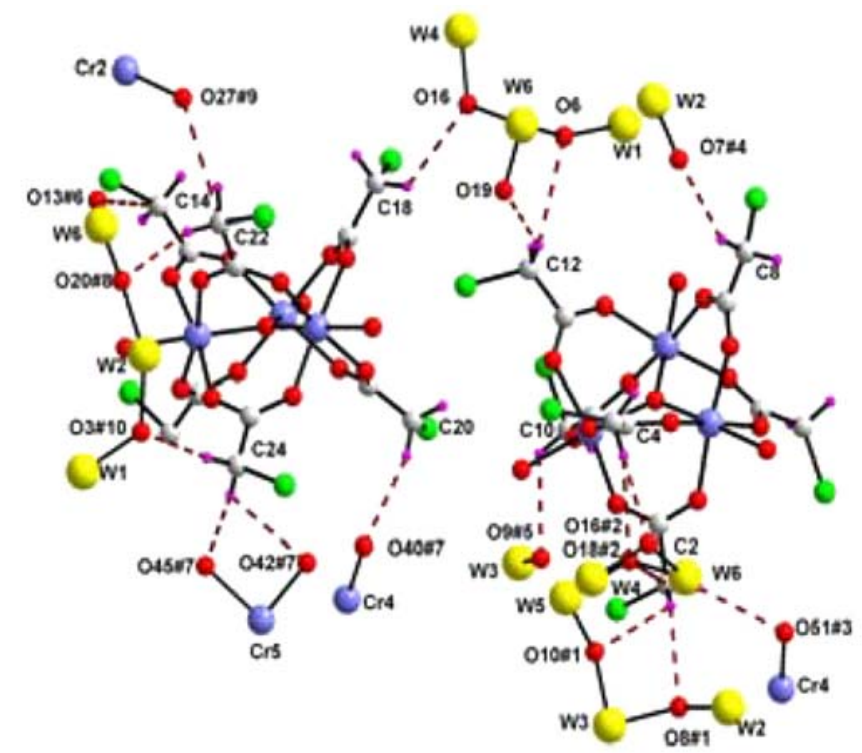

(b)

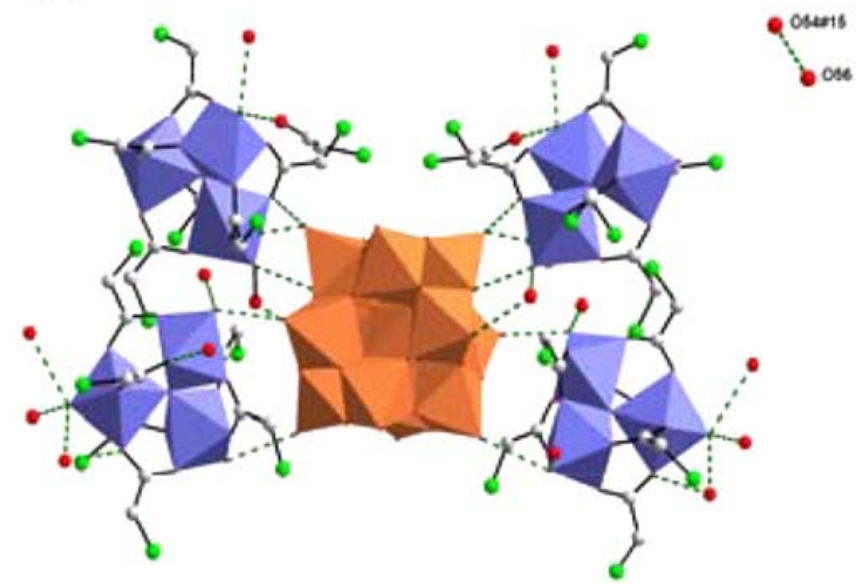

(c)

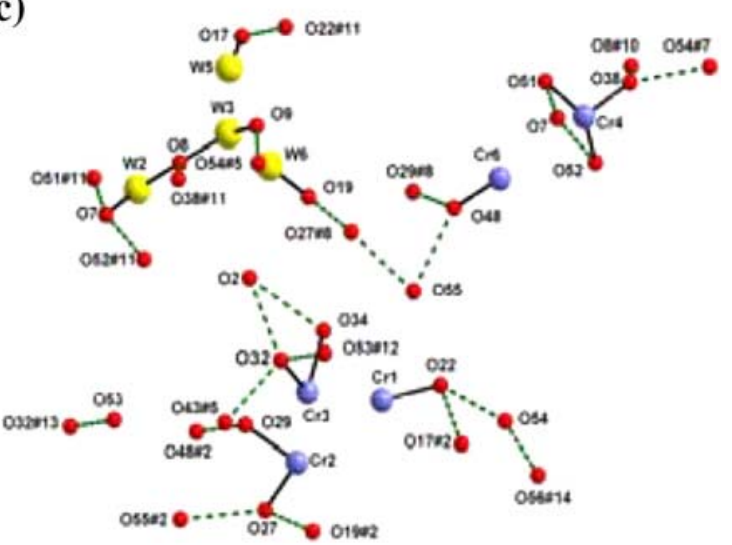

Figure 15. (a) $\mathrm{C}-\mathrm{H} \cdots \mathrm{O}$ hydrogen bonding interactions in $\left[\mathrm{Cr}_{3}\left(\mu_{3}-\mathrm{O}\right)\left(\mathrm{ClCH}_{2} \mathrm{COO}\right)_{6}\left(\mathrm{H}_{2} \mathrm{O}\right)_{3}\right]_{4}\left[\mathrm{SiW}_{12} \mathrm{O}_{40}\right] \cdot 8 \mathrm{H}_{2} \mathrm{O} \cdot 2 \mathrm{ClCH}_{2}$ $\mathrm{COOH}\left(\mathbf{2}^{\prime}\right)$ along with relevant symmetry code. (b) $\mathrm{O}-\mathrm{H} \cdots \mathrm{O}$ Hydrogen bonding interactions in compound $\mathbf{2}^{\prime}$. (c) $\mathrm{O}-\mathrm{H} \cdots \mathrm{O}$ Hydrogen bonding interactions with relevant symmetry codes in compound $\mathbf{2}^{\prime}$. Color code: W, yellow; $\mathrm{O}$, red; Cr, violet; $\mathrm{Cl}$, light green; $\mathrm{C}$, gray; $\mathrm{H}$, purple; $\left[\mathrm{Cr}_{3}\left(\mu_{3}-\mathrm{O}\right)\left(\mathrm{ClCH}_{2} \mathrm{COO}\right)_{6}\left(\mathrm{H}_{2} \mathrm{O}\right)_{3}\right]^{1+}$ cluster, violet polyhedra; $\left[\mathrm{SiW}_{12} \mathrm{O}_{40}\right]^{4-}$ cluster, golden yellow polyhedra; $\mathrm{C}-\mathrm{H} \cdots \mathrm{O}$ hydrogen bonding interactions, brown dotted lines; $\mathrm{O}-\mathrm{H} \cdots \mathrm{O}$ hydrogen bonding interactions green dotted lines.

at $85^{\circ} \mathrm{C}$ and $135^{\circ} \mathrm{C}$ for the period of 3.5 hours, they lose considerable number of lattice water molecules and generate the dehydrated analogues $\left[\mathrm{Fe}_{3}\left(\mu_{3}-\mathrm{O}\right)\left(\mathrm{ClCH}_{2} \mathrm{COO}\right)_{6}\right.$ $\left.\left(\mathrm{H}_{2} \mathrm{O}\right)_{3}\right]_{4}\left[\mathrm{SiW}_{12} \mathrm{O}_{40}\right] \cdot 10 \mathrm{H}_{2} \mathrm{O} \cdot 2 \mathrm{ClCH}_{2} \mathrm{COOH}\left(\mathbf{1}^{\prime}\right)$ and $\left[\mathrm{Fe}_{3}\left(\mu_{3}-\mathrm{O}\right)\left(\mathrm{ClCH}_{2} \mathrm{COO}\right)_{6}\left(\mathrm{H}_{2} \mathrm{O}\right)_{3}\right]_{4}\left[\mathrm{SiW}_{12} \mathrm{O}_{40}\right] \cdot 8 \mathrm{H}_{2} \mathrm{O}$. $2 \mathrm{ClCH}_{2} \mathrm{COOH}\left(\mathbf{1}^{\prime \prime}\right)$, respectively.

The dehydrated compounds $\mathbf{1}^{\prime}$ and $\mathbf{1}^{\prime \prime}$ are structurally characterized. The relevant crystal data and refinement parameters are given in the Table 1. Crystal structure of compounds $\mathbf{1}^{\prime}$ and $\mathbf{1}^{\prime \prime}$ contains ten and eight lattice water molecules per formula unit, respectively. The regular arrangement of lattice water molecules in hydrophilic channels (that include water dimer) of the crystal structure of compound $\mathbf{1}^{\prime}$ is depicted in Fig- ure 8(b). The less number of hydrogen bonded lattice water molecules compared to those in the parent compound $\mathbf{1}$ is expected in a dehydrated crystal. We have chosen $135^{\circ} \mathrm{C}$ as maximum temperature for reversible water exclusion experiment because if we heat the crystals beyond this temperature range, the pertinent crystals do not diffract in the X-ray diffractometer. The metal coordinated water molecules are supposed to leave at high temperature resulting in the formation of polycrystalline material. The release of lattice water molecules from 1 to $\mathbf{1}^{\prime \prime}$ leads to the shrinkage of the unit cell volume gradually as: $\mathbf{1}\left(\mathrm{V}=17106(8) \AA^{3}\right)>\mathbf{1}^{\prime}(\mathrm{V}=15084(7)$ $\left.\AA^{3}\right)>\mathbf{1}^{\prime \prime}\left(\mathrm{V}=14941(11) \AA^{3}\right)$. The shrinkage of unit cell, occurred in the crystallographic $c$ axis (see Table 1 
Table 12. Geometrical parameters of the $\mathrm{C}-\mathrm{H} \cdots \mathrm{O}$ hydrogen bonds $\left(\AA,{ }^{\circ}\right)$ involved in supramolecular network of compound $\left[\mathrm{Cr}_{3}\left(\mu_{3}-\mathrm{O}\right)\left(\mathrm{ClCH}_{2} \mathrm{COO}\right)_{6}\left(\mathrm{H}_{2} \mathrm{O}\right)_{3}\right]_{4}\left[\mathrm{SiW}_{12} \mathrm{O}_{40}\right] \cdot 8 \mathrm{H}_{2} \mathrm{O}$. $2 \mathrm{ClCH}_{2} \mathrm{COOH}\left(\mathbf{2}^{\prime}\right)$. $\mathrm{D}=$ donor; $\mathrm{A}=$ acceptor.

\begin{tabular}{|c|c|c|c|c|}
\hline $\mathrm{D}-\mathrm{H} \cdot \mathrm{A}$ & $\mathrm{d}(\mathrm{D} \cdots \mathrm{H})$ & $\mathrm{d}(\mathrm{H} \cdots \mathrm{A})$ & $\mathrm{d}(\mathrm{D} \cdots \mathrm{A})$ & $\angle$ (DHA \\
\hline C2-H2A....O8\#1 & 0.97 & 2.58 & $3.386(14)$ & 140.8 \\
\hline C2-H2A....O10\#1 & 0.97 & 2.77 & $3.440(15)$ & 127.0 \\
\hline C2-H2A....O18\#2 & 0.97 & 3.04 & $3.592(15)$ & 117.4 \\
\hline C2-H2B....O51\#3 & 0.97 & 2.56 & $3.352(14)$ & 138.3 \\
\hline C4-H4B....O16\#2 & 0.97 & 2.47 & $3.444(15)$ & 179.2 \\
\hline C4-H4B...O18\#2 & 0.97 & 2.98 & $3.597(16)$ & 122.6 \\
\hline C8-H8B....O7\#4 & 0.97 & 2.56 & $3.429(17)$ & 149.2 \\
\hline С10-H10A....O9\#5 & 0.97 & 2.57 & $3.222(15)$ & 124.7 \\
\hline C12-H12B....O6 & 0.97 & 2.75 & $3.391(14)$ & 123.8 \\
\hline C12-H12B....O19 & 0.97 & 2.30 & $3.116(16)$ & 140.8 \\
\hline C14-H14A....O13\#6 & 0.97 & 2.52 & $3.392(17)$ & 149.9 \\
\hline С18-H18A...O16 & 0.97 & 2.85 & $3.541(16)$ & 129.1 \\
\hline С20-H20A....O40\#7 & 0.97 & 2.57 & $3.500(2)$ & 162.5 \\
\hline C22-H22A....O20\#8 & 0.97 & 2.61 & $3.520(14)$ & 157.1 \\
\hline С22-H22B...O27\#9 & 0.97 & 2.75 & $3.337(14)$ & 119.5 \\
\hline С24-H24B...O3\#10 & 0.97 & 2.43 & $3.383(14)$ & 168.6 \\
\hline С24-H24B...O42\#7 & 0.97 & 2.81 & $3.443(16)$ & 123.2 \\
\hline C24-H24A...O45\#7 & 0.97 & 2.56 & $3.518(16)$ & 171.5 \\
\hline
\end{tabular}

Symmetry transformations used to generate equivalent atoms: \#1 $0.5+\mathrm{x},-0.5+\mathrm{y}, \mathrm{z}$; \#2 0.5-x, -0.5+y, 0.5-z; \#3 x, -1+y, z; \#4 1-x, y, 0.5-z; \#5 0.5-x, 0.5-y, -z; \#6 0.5-x, 1.5-y, -z; \#7 -x, 1-y, -z; \#8 0.5-x, 0.5+y, 0.5-z; \#9 x, 1+y, z; \#10 -0.5+x, 0.5+y, z; \#11 0.5+x, -0.5+y, z; \#12 x, y, -1+z; \#13 x, y, 1+z; \#14 -1+x, y, z; \#15 1+x, y, z.

Table 13. Geometrical parameters of the $\mathrm{O}-\mathrm{H} \cdots \mathrm{O}$ hydrogen bonds $(\AA)$ involved in supramolecular network of compound dehydrated $2-85^{\circ}$.

\begin{tabular}{lccc}
\hline $\mathrm{O}(7)-\mathrm{O}(51) \# 11$ & $2.919(11)$ & $\mathrm{O}(7)-\mathrm{O}(52) \# 11$ & $3.006(11)$ \\
$\mathrm{O}(8)-\mathrm{O}(38) \# 11$ & $2.765(10)$ & $\mathrm{O}(9)-\mathrm{O}(54) \# 5$ & $3.073(13)$ \\
$\mathrm{O}(17)-\mathrm{O}(22) \# 8$ & $2.905(11)$ & $\mathrm{O}(19)-\mathrm{O}(27) \# 8$ & $2.736(10)$ \\
$\mathrm{O}(22)-\mathrm{O}(17) \# 2$ & $2.905(11)$ & $\mathrm{O}(22)-\mathrm{O}(54)$ & $2.717(12)$ \\
$\mathrm{O}(27)-\mathrm{O}(19) \# 2$ & $2.736(10)$ & $\mathrm{O}(27)-\mathrm{O}(55) \# 2$ & $2.793(13)$ \\
$\mathrm{O}(29)-\mathrm{O}(48) \# 2$ & $2.913(12)$ & $\mathrm{O}(32)-\mathrm{O}(2)$ & $2.856(11)$ \\
$\mathrm{O}(32)-\mathrm{O}(43) \# 5$ & $3.049(13)$ & $\mathrm{O}(32)-\mathrm{O}(53) \# 12$ & $2.540(2)$ \\
$\mathrm{O}(34)-\mathrm{O}(2)$ & $3.036(11)$ & $\mathrm{O}(38)-\mathrm{O}(8) \# 10$ & $2.765(10)$ \\
$\mathrm{O}(38)-\mathrm{O}(54) \# 7$ & $2.750(12)$ & $\mathrm{O}(48)-\mathrm{O}(29) \# 8$ & $2.913(12)$ \\
$\mathrm{O}(48)-\mathrm{O}(55)$ & $2.795(13)$ & $\mathrm{O}(51)-\mathrm{O}(7) \# 10$ & $2.919(11)$ \\
$\mathrm{O}(52)-\mathrm{O}(7) \# 10$ & $3.006(11)$ & $\mathrm{O}(53)-\mathrm{O}(32) \# 13$ & $2.540(2)$ \\
$\mathrm{O}(54)-\mathrm{O}(56) \# 14$ & $2.735(18)$ & $\mathrm{O}(55)-\mathrm{O}(27) \# 8$ & $2.793(13)$ \\
$\mathrm{O}(56)-\mathrm{O}(54) \# 15$ & $2.735(18)$ & & \\
\hline
\end{tabular}

Symmetric codes are already specified in the footnote of Table 12 .

for unit cell data), confirms that the water molecules accommodated in the crystallographic $c$ axis are readily released from the crystal lattice.

The packing diagrams of compounds $\mathbf{1}$ and $\mathbf{1}^{\prime}$ viewed down the crystallographic $b$ axis are presented in Figure 10. For an assessment of the unit cell volume shrinkage, the corresponding internuclear distances between two Keggin anion clusters (for compounds $\mathbf{1}$ and $\mathbf{1}^{\prime}$ ) along the $c$ axis are measured and represented in Table 4. The $\mathrm{C}-\mathrm{H} \cdots \mathrm{O}$ and $\mathrm{O}-\mathrm{H} \cdots \mathrm{O}$ hydrogen bonding interactions, observed in the crystal structure of compound $\mathbf{1}^{\prime}$, are shown in Figure 11 and the relevant parameters are given in Tables 5 and 6 , respectively. 
Likewise, in compound $\mathbf{1}^{\prime \prime}, \mathrm{C}-\mathrm{H} \cdots \mathrm{O}$ and $\mathrm{O}-\mathrm{H} \cdots \mathrm{O}$ hydrogen bonding interactions are shown in Figure 12 and appropriate parameters are presented in Tables 7 and 8 , respectively. Crystals of compounds 1' (heated compound) on exposure to water vapor at ambient condition for two weeks, get moistened without their dissolution and reverts back to the parent compound, regenerated 1. The color of the heated crystals (light red) became dark red after exposing to the water vapor. A single crystal with suitable size from this water regenerated bunch of the crystals has been mounted on the single crystal X-ray diffractometer for data collection. Reentering of the water molecules in the crystal lattice leads to expansion of the unit cell volume as: $\mathbf{1}^{\prime}(\mathrm{V}=$ $\left.15084.00 \AA^{3}\right)<\operatorname{regenerated} 1\left(\mathrm{~V}=17039 \AA^{3}\right)$. The obtained cell data for compound regenerated 1 exactly match to that of parent compound 1; however, we have not succeeded to solve the crystal structure of regenerated 1 because of the poor quality of reflection data.

$3.6\left[\mathrm{Cr}_{3}\left(\mu_{3}-\mathrm{O}\right)\left(\mathrm{ClC} \mathrm{H}_{2} \mathrm{COO}\right)_{6}\left(\mathrm{H}_{2} \mathrm{O}\right)_{3}\right]_{4}$ $\left[\mathrm{Si} \mathrm{W}_{12} \mathrm{O}_{40}\right] \cdot 14 \mathrm{H}_{2} \mathrm{O} \cdot 2 \mathrm{ClCH}_{2} \mathrm{COOH}(\mathbf{2})$

Dark green colored crystals of compound $\mathbf{2}$ were grown from an acidic aqueous medium containing $\mathrm{H}_{4} \mathrm{SiW}_{12} \mathrm{O}_{40}, \mathrm{ClCH}_{2} \mathrm{COOH}$ and $\mathrm{Cr}(\mathrm{NO})_{3} \cdot 9 \mathrm{H}_{2} \mathrm{O}$. Compound 2 crystallizes in monoclinic system with space group $C 2 / c$ and single crystal X-ray data obtained at 298 $\mathrm{K}$ are summarized in Table 9 . The pertinent asymmetric unit contains half part of Keggin anion (in which Si atom is in half occupancy), two basic trinuclear chromiumoxo macrocations, one lattice $\mathrm{ClCH}_{2} \mathrm{COOH}$ and seven non-coordinated water molecules. Thermal ellipsoidal plot of compound $\left.\mathrm{Cr}_{3}\left(\mu_{3}-\mathrm{O}\right)\left(\mathrm{ClCH}_{2} \mathrm{COO}\right)_{6}\left(\mathrm{H}_{2} \mathrm{O}\right)_{3}\right]_{4}$ $\left[\mathrm{SiW}_{12} \mathrm{O}_{40}\right] \cdot 14 \mathrm{H}_{2} \mathrm{O} \cdot 2 \mathrm{ClCH}_{2} \mathrm{COOH}(2)$ with atom labeling scheme is presented in Figure 13.

Compound $\mathbf{2}$ is isostructural to compound $\mathbf{1}$ and hence coordination bonding environments around metal centers are similar. In the crystal structure, two noncoordinated $\mathrm{ClCH}_{2} \mathrm{COOH}$ were located in the lattice and those were refined isotropically due to considerable disorder. Crystal structure of compound 2 contains fourteen lattice water molecules. A water dimer, exclusively formed by lattice water molecules, O53 and $\mathrm{O} 54$, is crytallographically characterized. The O$\mathrm{H}$. . . O hydrogen bonding distance is 2.68(5) A. Overall, $\mathrm{C}-\mathrm{H} \cdots \mathrm{O}$ and $\mathrm{O}-\mathrm{H} \cdots \mathrm{O}$ hydrogen bonding interactions are shown in Figure 14. The relevant hydrogen bonding parameters are presented in Tables 10 and 11, respectively.

\subsection{Water exclusion (dehydration) and inclusion (regeneration) experiments in compound 2}

The similar heating experiments were repeated for compound $\mathbf{2}$ as performed with compound $\mathbf{1}$. Heating the bunch of crystals of compound 2 at $85^{\circ} \mathrm{C}$ and $135^{\circ} \mathrm{C}$ for the period of 3.5 hours loses considerable number of lattice water molecules and generate corresponding dehydrated analogues $\left[\mathrm{Cr}_{3}\left(\mu_{3}-\right.\right.$ $\left.\mathrm{O})\left(\mathrm{ClCH}_{2} \mathrm{COO}\right)_{6}\left(\mathrm{H}_{2} \mathrm{O}\right)_{3}\right]_{4}\left[\mathrm{SiW}_{12} \mathrm{O}_{40}\right] \cdot 8 \mathrm{H}_{2} \mathrm{O} \cdot 2 \mathrm{ClCH}_{2}$ $\mathrm{COOH}\left(\mathbf{2}^{\prime}\right)$.

The dehydrated compounds dehydrated $\mathbf{2 - 8 5}^{\mathbf{0}}\left(\mathbf{2}^{\prime}\right)$ and dehydrated $2-\mathbf{1 3 5}^{\circ}\left(\mathbf{2}^{\prime}\right)$ were structurally characterized and crystal data and refinement parameters are presented in the Table 9. The $\mathrm{C}-\mathrm{H} \cdots \mathrm{O}$ and $\mathrm{O}-\mathrm{H} \cdots \mathrm{O}$ hydrogen bonding interactions observed in the crystal structure of compound $\mathbf{2}^{\prime}$ is shown in Figure 15 and the relevant hydrogen bonding parameters are given in Tables 12 and 13, respectively. The release of lattice water molecules (water exclusion) from $\mathbf{2}$ to $\mathbf{2}^{\prime}$ leads to the shrinkage of the unit cell volume as follows: $\mathbf{2}$ $\left(\mathrm{V}=16907(16) \AA^{3}\right)>\mathbf{2}^{\prime}\left(\mathrm{V}=14936(5) \AA^{3}\right)$.

When dehydrated compound $\mathbf{2}^{\prime}$ is exposed to water vapor for the period of one week, it regenerates the parent compound 2 (regenerated 2). The unit cell parameter of water regenerated compound (regenerated 2) is as follows: $a=30.319 \AA, b=17.655$ $\AA, c=32.771 \AA$ and $\beta=102.588^{\circ}, \mathrm{V}=17120.53$ $\AA^{3}$. The obtained unit cell volume exactly matches to the parent compound 2 , which clearly indicates the expansion of unit cell as, dehydrated $2^{\prime}(\mathrm{V}=14936(5)$ $\left.\AA^{3}\right)<\operatorname{regenerated} 2\left(\mathrm{~V}=17120.53 \AA^{3}\right)$. Although we could obtain the unit cell parameters of the water regenerated compound by X-ray diffractometry, the corresponding structure could not be solved because of the poor quality of reflection data.

\section{Conclusions}

In summary, we have synthesized compounds $\mathbf{1}$ and $\mathbf{2}$ and we have generated their dehydrated forms. We have characterized them unambiguously by single crystal Xray structure determination. The rehydrated compounds have been characterized by determination of their unit cell parameters. The solid state properties of compound $\mathbf{1}$ and $\mathbf{1}^{\prime}$ are described in the view of exclusion (shrinkage of unit cell volume) and inclusion (expansion of unit cell volume) of water molecules in the crystal lattice. This phenomenon can be described as breathing of the crystals. We report here the first example of 'ionic crystal' in which macrocation/polyoxometalate ratio is $4: 1$, without the presence of alkali metal ions. 
To the best of our knowledge, 4:1 is the highest trinuclear macrocation/polyoxoanion ratio observed in the crystal structure. The supramolecular $\mathrm{O}-\mathrm{H}$. . O and $\mathrm{C}-\mathrm{H}$. . O hydrogen bonding interactions of all the compounds are discussed elaborately. The adsorption and desorption properties of trinuclear macrocation and polyoxometalate based ion pair complexes systems will be an interesting study. The macrocations with similar coordination functionalities will be prepared and corresponding adsorption properties of ionic crystal and their related studies will be performed in near future.

\section{Supplementary Information (SI)}

CCDC 1541077, CCDC 1541078, CCDC 1541084, CCDC 1541100 and CCDC 1541105 contain the supplementary crystallographic data for compounds $\mathbf{1}, \mathbf{1}^{\prime}, \mathbf{1}^{\prime \prime}, \mathbf{2}$ and $\mathbf{2}^{\prime}$, respectively. Relevant crystal data can be obtained free of charge via http://www.ccdc.cam.atc.uk/conts/retrieving. html, or from the Cambridge Crystallographic Data Centre, 12 Union Road, Cambridge CB2 1EZ, UK; fax: (+44) 1223336- 033; or e-mail: deposit@ccdc.cam.ac.uk.

\section{Acknowledgements}

We thank SERB, DST, Government of India, for financial support (Project No. SB/S1/IC-34/2013). We are grateful to UGC, New Delhi, for UPE-II grant. We acknowledge DST PURSE and FIST grants.

\section{References}

1. Vlachos A, Psycharis V, Raptopoulou C P, Lalioti N, Sanakis Y, Diamantopoulos G, Fardis M, Karayanni M, Papavassiliou G and Terzis A 2004 A nearly symmetric trinuclear chromium(III) oxo carboxylate assembly: preparation, molecular and crystal structure, and magnetic properties of $\left[\mathrm{Cr}_{3} \mathrm{O}\left(\mathrm{O}_{2} \mathrm{CPh}\right)_{6}(\mathrm{MeOH})_{3} 2 \mathrm{MeOH}\right.$ Inorg. Chim. Acta 3573162

2. Fujihara T, Aonahata J, Kumakura S, Nagasawa A, Murakami K and Ito T 1998 Kinetic Study on the Substitution of Dimethylacetamide for the Terminal Aqua Ligands in the Trinuclear Chromium(III) Complexes $\left[\mathrm{Cr}_{3}\left(\mu_{3}-\mathrm{O}\right)\left(\mu-R \mathrm{CO}_{2}\right) 6\left(\mathrm{H}_{2} \mathrm{O}\right)_{3}\right]^{+}(\mathrm{R}) \mathrm{H}$, $\mathrm{CH}_{3}, \mathrm{CH}_{3} \mathrm{CH}_{2}, \mathrm{CH}_{2} \mathrm{Cl}, \mathrm{CHCl}_{2}, \mathrm{CH}_{3} \mathrm{OCH}_{2},\left(\mathrm{CH}_{3}\right)_{3} \mathrm{C}$, $\left.\mathrm{CH}_{2} \mathrm{ClCH}_{2},\left(\mathrm{CH}_{3} \mathrm{CH}_{2}\right)_{2} \mathrm{CH}\right)$. Elucidation of the Mechanism from the Activation Volumes and the Substituent Effects of Bridging Carboxylate Ligands Inorg. Chem. 373779

3. Anson C E, Bourke J P, Cannon R D, Jayasooriya U A, Molinier M and Powell A K 1997 Crystal Structures of the Isomorphous Prototypic Oxo-Centered Trinuclear Complexes $\left[\mathrm{Cr}_{3} \mathrm{O}\left(\mathrm{OOCCH}_{3}\right)_{6}\left(\mathrm{H}_{2} \mathrm{O}\right)_{3}\right] \mathrm{Cl} \cdot 6 \mathrm{H}_{2} \mathrm{O}$ and $\left[\mathrm{Fe}_{3} \mathrm{O}\left(\mathrm{OOCCH}_{3}\right)_{6}\left(\mathrm{H}_{2} \mathrm{O}\right)_{3}\right] \mathrm{Cl} \cdot 6 \mathrm{H}_{2} \mathrm{O}$ Inorg. Chem. 36 1265

4. Cannon R D, Jayasooriya U A, Sowrey F E, Tilford C, Little A, Bourke J P, Rogers R D, Vincent
J B and Kearley G J 1998 Concealed Asymmetry in an Exchange-Coupled Trichromium(III) Cluster: Structure and Magnetic Spectrum of $\left[\mathrm{Cr}_{3} \mathrm{O}(\mathrm{OOCPh})_{6}(\mathrm{py})_{3}\right](\mathrm{py})_{0.5} \mathrm{ClO}_{4}$ Inorg. Chem. 375675

5. Keeney L and Hynes M J 2005 Kinetics and mechanisms of the electron transfer reactions of oxo-centred carboxylate bridged complexes, $\left[\mathrm{Fe}_{3}\left(\mu_{3}-O\right)\left(\mathrm{O}_{2} \mathrm{CR}\right)_{6} \mathrm{~L}_{3}\right] \mathrm{ClO}_{4}$, with verdazyl radicals in acetonitrile solution Dalton Trans. 1524

6. Cannon R D and White R P 1988 In Progress in Inorganic Chemistry Stephen J Lippard (Ed.) vol. 36 pp. 195-298

7. Palii S P, Richardson D E, Hansen M L, Iversen B B, Larsen F K, Singerean L, Timco G A, Gerbeleu N V, Jennings K R and Eyler J R 2001 Mixed-terminal-ligand oxo-centered carboxylate-bridged trinuclear complexes: gas phase generation by means of electrospray ionization FT-ICR MS, condensed phase synthesis, and X-ray structure of $\mathrm{K}^{+}\left[\mathrm{Cr}_{3} \mathrm{O}\left(\mathrm{C}_{6} \mathrm{H}_{5} \mathrm{COO}\right)_{6}(\mathrm{~F})_{2}\left(\mathrm{H}_{2} \mathrm{O}\right)\right]^{-}$. $2\left(\mathrm{CH}_{3}\right)_{2} \mathrm{CO}$ Inorg. Chim. Acta 31923

8. Sowrey F E, Tilford C, Wocadlo S, Anson C E, Powell A K, Bennington S M, Montfrooij W, Jayasooriya U A and Cannon R D 2001 Spin frustration and concealed asymmetry: structure and magnetic spectrum of $\left[\mathrm{Fe}_{3} \mathrm{O}\left(\mathrm{O}_{2} \mathrm{CPh}\right)_{6}(\mathrm{py})_{3}\right] \mathrm{ClO}_{4} \cdot$ py Dalton Trans. 862

9. Murao T 1974 Jahn-Teller effect in trinuclear complexes Phys. Lett. 49A 33

10. Supriya S and Das S K 2007 Reversible Single Crystal to Single Crystal Transformation through $\mathrm{Fe}-\mathrm{O}(\mathrm{H}) \mathrm{Me} / \mathrm{Fe}-$ $\mathrm{OH}_{2}$ Bond Formation/Bond Breaking in a Gas-Solid Reaction at an Ambient Condition J. Am. Chem. Soc. 1293464

11. Supriya S and Das S K 2015 Reversible solid to solid transformation in a crystalline state gas-solid reaction under ambient conditions: $\mathrm{Fe}-\mathrm{N}$ (pyridine) bond formation at the expense of $\mathrm{Fe}-\mathrm{OH}_{2}$ bond breaking and vice versa CrystEngComm 178850

12. Gorun S M and Lippard S J 1986 A new synthetic approach to the ferritin core uncovers the soluble iron(III) oxo-hydroxo aggregate $\left[\mathrm{Fe}_{11} \mathrm{O}_{6}(\mathrm{OH})_{6}\left(\mathrm{O}_{2} \mathrm{CPh}\right)_{15}\right]$ Nature 319666

13. Gorun S M, Papaefthymiou G C, Frankel R B and Lippard S J 1987 Synthesis, Structure, and Properties of an Undecairon(III) Oxo-Hydroxo Aggregate: An Approach to the Polyiron Core in Ferritin J. Am. Chem. Soc. 109 3337

14. Welo L A 1928 Magnetic studies on salts, with particular reference to those with complex ions Philos. Mag. 6481

15. Toma H E, Araki K, Alexiou A D P, Nikolaou S and Dovidauskas S 2001 Monomeric and extended oxo-centered triruthenium clusters Coord. Chem. Rev. 219-221 187

16. Sudik A C, Côté A P, Wong-Foy A G, O'Keeffe M and Yaghi O M 2006 A Metal Organic Framework with a Hierarchical System of Pores and Tetrahedral Building Blocks Angew. Chem. Int. Ed. $\mathbf{4 5} 2528$

17. Uchida S, Kawamoto R and Mizuno N 2006 Recognition of Small Polar Molecules with an Ionic Crystal of $\alpha$-Keggin-Type Polyoxometalate with a Macrocation Inorg. Chem. $\mathbf{4 5} 5136$

18. Mizuno N and Uchida S 2006 Structures and Sorption Properties of Ionic Crystals of Polyoxometalates with Macrocation Chem. Lett. 35688 
19. Uchida S, Hashimoto M and Mizuno N 2002 A Breathing Ionic Crystal Displaying Selective Binding of Small Alcohols and Nitriles: $\mathrm{K}_{3}\left[\mathrm{Cr}_{3} \mathrm{O}(\mathrm{OOCH})_{6}\left(\mathrm{H}_{2} \mathrm{O}\right)_{3}\right][\alpha-$ $\left.\mathrm{SiW}_{12} \mathrm{O}_{40}\right] \cdot 16 \mathrm{H}_{2} \mathrm{O}$ Angew. Chem. Int. Ed. 412814

20. Uchida S and Mizuno N 2003 Unique GuestInclusion Properties of a Breathing Ionic Crystal of $\mathrm{K}_{3}\left[\mathrm{Cr}_{3} \mathrm{O}(\mathrm{OOCH})_{6}\left(\mathrm{H}_{2} \mathrm{O}\right)_{3}\right]\left[\alpha-\mathrm{SiW}_{12} \mathrm{O}_{40}\right]$. $16 \mathrm{H}_{2} \mathrm{O}$ Chem. Eur. J. 95850

21. Uchida $\mathrm{S}$ and Mizuno N 2004 Zeotype Ionic Crystal of $\mathrm{Cs}_{5}\left[\mathrm{Cr}_{3} \mathrm{O}(\mathrm{OOCH})_{6}\left(\mathrm{H}_{2} \mathrm{O}\right)_{3}\right]\left[\alpha-\mathrm{CoW}_{12} \mathrm{O}_{40}\right] \cdot 7.5 \mathrm{H}_{2} \mathrm{O}$ with Shape-Selective Adsorption of Water J. Am. Chem. Soc. 1261602

22. Uchida S, Kawamoto R, Akatsuka T, Hikichi S and Mizuno N 2005 Structures and Sorption Properties of Ionic Crystals of Macrocation-Dawson-Type Polyoxometalates with Different Charges Chem. Mater. 171367

23. Kawamoto R, Uchida S and Mizuno N 2005 Amphiphilic Guest Sorption of $\mathrm{K}_{2}\left[\mathrm{Cr}_{3} \mathrm{O}\left(\mathrm{OOCC}_{2} \mathrm{H}_{5}\right)_{6}\right.$ $\left.\left(\mathrm{H}_{2} \mathrm{O}\right)_{3}\right]_{2}\left[\alpha-\mathrm{SiW}_{12} \mathrm{O}_{40}\right]$ Ionic Crystal J. Am. Chem. Soc. 12710560

24. Ogasawara Y, Uchida S and Mizuno N 2007 States of Water in Ionic Crystals of States of Water in Ionic Crystals of $\left[\mathrm{Cr}_{3} \mathrm{O}(\mathrm{OOCH})_{6}\left(\mathrm{H}_{2} \mathrm{O}\right)_{3}\right]^{+}$Macrocation with $\alpha$-Keggin-Type Polyoxometalates J. Phys. Chem. C 111 8218

25. Lesbani A, Kawamoto R, Uchida S and Mizuno N 2008 Control of Structures and Sorption Properties of Ionic Crystals of $\mathrm{A}_{2}\left[\mathrm{Cr}_{3} \mathrm{O}\left(\mathrm{OOCC}_{2} \mathrm{H}_{5}\right)_{6}\left(\mathrm{H}_{2} \mathrm{O}\right)_{3}\right]_{2}[\alpha-$ $\mathrm{SiW}_{12} \mathrm{O}_{40}$ ] $\left(\mathrm{A}=\mathrm{Na}, \mathrm{K}, \mathrm{Rb}, \mathrm{NH}_{4}, \mathrm{Cs}\right.$, TMA) Inorg. Chem. 473349

26. Rocchiccioli-Deltcheff C, Fournier M, Franck R and Thouvenot R 1983 Vibrational Investigations of Poly- oxometalates. 2. Evidence for Anion-Anion Interactions in Molybdenum(V1) and Tungsten(V1) Compounds Related to the Keggin Structure Inorg. Chem. 22 207

27. SAINT 1998 Software for the CCD Detector System (Bruker Analytical X-ray Systems Inc: Madison WI USA)

28. Sheldrick G M 1997 SHELXS-97program for Solution of Crystal Structures (University of Göttingen: Göttingen)

29. Sheldrick G M 1997 SHELXL-97 Program for Refinement of Crystal Structures (University of Göttingen: Göttingen)

30. Brandenburg K 2001 DIAMOND Version 2.1e, Crystal Impact GbR, Bonn, Germany

31. Nozaki C, Kiyoto I, Minai $\mathrm{Y}$, Misono $\mathrm{M}$ and Mizuno N 1999 Synthesis and Characterization of Diiron(III)-Substituted Silicotungstate, $[\gamma(1,2)$ $\left.\mathrm{SiW}_{10}\left\{\mathrm{Fe}\left(\mathrm{OH}_{2}\right)\right\}_{2} \mathrm{O}_{38}\right]^{6-}$ Inorg. Chem. 385724

32. Zonnevijlle F, Tourné C M and Tourné G F 1982 Preparation and Characterization of Iron(II1)- and Rhodium(II1)-Containing Heteropolytungstates. Identification of Novel Oxo-Bridged Iron(111) Dimers Inorg. Chem. 212751

33. Supriya S, Manikumari S, Ragahvaiah $\mathrm{P}$ and Das $\mathrm{S}$ K 2003 A cyclic supramolecular $\left(\mathrm{H}_{2} \mathrm{O}\right)_{4}$ cluster in an unusual $\mathrm{Fe}_{3}$ complex that aggregates to $\{\mathrm{Fe} 3\}_{n}$ with a zig-zag chainlike structure New J. Chem. 27218.

34. Supriya S and Das S K 2003 A cyclic $\left(\mathrm{H}_{2} \mathrm{O}\right) 4$ cluster characterized in the solid state disappears on heating and regenerates from water vapor: A supramolecular reversible gas-solid reaction New J. Chem. 27 1568 